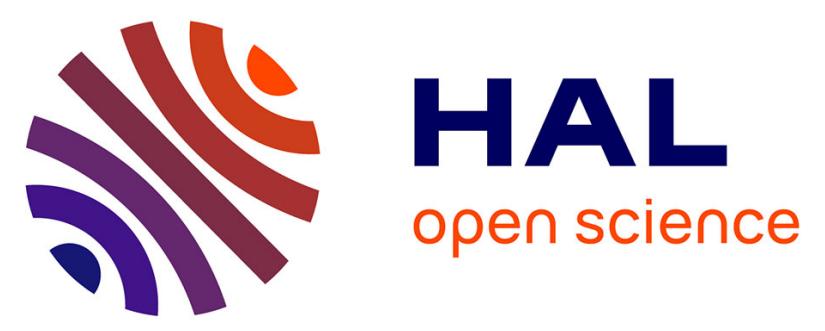

\title{
The minor pilin PilV provides a conserved adhesion site throughout the antigenically variable meningococcal type IV pilus
}

\author{
Jean-Philippe Barnier, Julie Meyer, Subramania Kolappan, Haniaa \\ Bouzinba-Ségard, Gaël Gesbert, Anne Jamet, Eric Frapy, Sophia \\ Schönherr-Hellec, Elena Capel, Zoé Virion, et al.
}

\section{To cite this version:}

Jean-Philippe Barnier, Julie Meyer, Subramania Kolappan, Haniaa Bouzinba-Ségard, Gaël Gesbert, et al.. The minor pilin PilV provides a conserved adhesion site throughout the antigenically variable meningococcal type IV pilus. Proceedings of the National Academy of Sciences of the United States of America, 2021, 118 (45), pp.e2109364118. 10.1073/pnas.2109364118 . hal-03552815

\section{HAL Id: hal-03552815 \\ https://hal.science/hal-03552815}

Submitted on 15 Feb 2022

HAL is a multi-disciplinary open access archive for the deposit and dissemination of scientific research documents, whether they are published or not. The documents may come from teaching and research institutions in France or abroad, or from public or private research centers.
L'archive ouverte pluridisciplinaire HAL, est destinée au dépôt et à la diffusion de documents scientifiques de niveau recherche, publiés ou non, émanant des établissements d'enseignement et de recherche français ou étrangers, des laboratoires publics ou privés. 
1 THE MINOR PILIN PILV PROVIDES A CONSERVED ADHESION SITE

2 THROUGHOUT THE ANTIGENICALLY VARIABLE MENINGOCOCCAL TYPE IV

3 PILUS

4 Jean-Philippe Barnier ${ }^{1,2,3}$, Julie Meyer $^{1,2, *}$, Subramania Kolappan ${ }^{4, *}$, Haniaa Bouzinba-

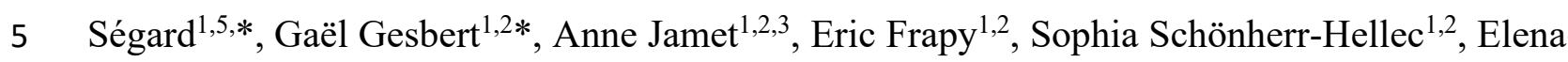

6 Capel $^{1,2}$, Zoé Virion ${ }^{1,2}$, Marion Dupuis ${ }^{1,2}$, Emmanuelle Bille ${ }^{1,2,3}$, Philippe Morand ${ }^{1,2,6}$, Taliah

7 Schmitt $^{7}$, Sandrine Bourdoulous ${ }^{1,5}$, Xavier Nassif $^{1,2,3}$, Lisa Craig $^{4, \dagger}$ and Mathieu Coureuil ${ }^{1,2, \dagger}$

8

9 1. Université de Paris, Faculté de Médecine, Paris, France,

2. Institut Necker Enfants-Malades, Inserm U1151, CNRS UMR 8253, Paris, France,

3. Service de microbiologie, Assistance Publique-Hôpitaux de Paris. Centre-Université de Paris, hôpital Necker Enfants Malades, Paris, France

4. Department of Molecular Biology and Biochemistry, Simon Fraser University, Burnaby BC

Canada, V5A 3 Y6.

5. Institut Cochin, Inserm U1016, CNRS UMR 8104, Paris, France

6. Service de Bactériologie, Assistance Publique-Hôpitaux de Paris. Centre-Université de Paris, hôpital Cochin, Paris, France.

7. Service de chirurgie reconstructrice et plastique, Groupe Hospitalier Paris Saint-Joseph, Paris, France

$21 *$ equal contribution

$22+$ Correspondence to lisa_craig@sfu.ca or mathieu.coureuil@inserm.fr 


\section{ABSTRACT}

Neisseria meningitidis utilizes type IV pili to adhere to and colonize host endothelial cells, a process at the heart of meningococcal invasive diseases leading to meningitis and sepsis. Type IV pili are polymers of an antigenically variable major pilin building block, PilE, plus several core minor pilins that initiate pilus assembly and are thought to be located at the pilus tip. Adhesion of $N$. meningitidis to human endothelial cells requires both PilE and a conserved noncore minor pilin PilV but the localization of PilV and its precise role in this process remains to be clarified. Here we show that both PilE and PilV promote adhesion to endothelial vessels in vivo. The substantial adhesion defect observed for pilV mutants suggests it is the main adhesin. Consistent with this observation, super-resolution microscopy showed its abundant distribution throughout the pilus. We determined the crystal structure of PilV and modeled in within the pilus filament. The small size of PilV causes it to be recessed relative to adjacent PilE subunits, which are dominated by a prominent hypervariable loop. Nonetheless, we identified a conserved surface-exposed adhesive loop on PilV by alanine scanning mutagenesis. Critically, antibodies directed against PilV inhibit $N$. meningitidis colonization of human skin grafts. These findings explain how $N$. meningitidis T4P undergo antigenic variation to evade the humoral immune response while maintaining their adhesive function, and establish the potential of this highly conserved minor pilin as a vaccine and therapeutic target for the prevention and treatment of $N$. meningitidis infections. 
43 Type IV pili (T4P) are among the most widespread adhesive factors in prokaryotes. In 44 pathogenic Neisseria the major pilin, which provides the structural framework for the 45 filamentous T4P, undergoes antigenic variation allowing the bacteria to evade the humoral 46 immune response without impacting host cell adhesion. Here we show that a minor pilin, PilV, 47 is distributed throughout the pilus and contributes to Neisseria meningitidis adhesion, and that 48 antibodies to PilV block meningococcal adhesion in vivo. Our results provide a mechanism 49 whereby N. meningitidis varies its immunodominant major pilin to escape antibody recognition 50 while maintaining conserved sites throughout the pilus for host receptor binding. They further 51 suggest a strategy to prevent or block deadly N. meningitidis infections by targeting this minor 52 pilin. 


\section{INTRODUCTION}

The human-restricted bacterial pathogen Neisseria meningitidis is a leading cause of meningitis and sepsis worldwide and represents a significant global public health threat (Chang et al., 2012; Muttalif et al., 2019). N. meningitidis is carried asymptomatically in the protective mucus layer of the throat for $5 \%$ to $25 \%$ of the population (Christensen et al., 2010; Coureuil et al., 2019; Audry et al., 2019). In some cases, N. meningitidis disseminates into the bloodstream, an environment to which this bacterium is remarkably well-adapted. Meningococci possess a polysaccharide capsule that protects them against complement deposition, plus several membrane associated factors that are important for survival, including factor $\mathrm{H}$ binding protein and iron uptake systems (Siena et al., 2018). Critical to $N$. meningitidis survival in the bloodstream are the type IV pili (T4P), which mediate vascular colonization; non-piliated meningococci are rapidly cleared from the blood (Barnier et al., 2021; Capel et al., 2017; Denis et al., 2019). T4P are long filamentous appendages displayed peritrichously on the bacterium. The major pilin protein, PilE, is the primary building block of the pilus. This and other surfacedisplayed $N$. meningitidis proteins undergo antigenic variation, allowing this pathogen to evade a protective immune response (Cahoon and Seifert, 2009; Vink et al., 2012; Wörmann et al., 2014).

T4P are responsible for acute colonization of human blood vessels and are thus essential in establishing invasive meningococcal diseases (Barnier et al., 2021; Bernard et al., 2014; Denis et al., 2019; Join-Lambert et al., 2013). T4P are helical polymers of the major pilin assembled by the T4P machinery (Craig et al., 2019; McCallum et al., 2019). The conserved $\mathrm{N}$-terminus of the major pilin is a hydrophobic $\alpha$-helix that tethers the C-terminal globular domain in the inner membrane prior to pilus assembly, and forms a helical array in the core of the intact pilus, displaying the globular domain on the filament surface. Pilus assembly is initiated by a cluster of pilin-like proteins called minor pilins (Nguyen et al., 2015; Kolappan 
et al., 2015; Ng et al., 2016; Treuner-Lange et al., 2020). These "core" minor pilins are thought to localize to the pilus tip. The major pilin, PilE, is highly conserved in amino acid sequence and structure between N. meningitidis and the urogenital pathogen Neisseria gonorrhoeae with the exception of a hypervariable $\beta$-hairpin near the C-terminus that is prominent on the pilus surface (Parge et al., 1995; Craig et al., 2006; Kolappan et al., 2016; Wang et al., 2017). In N. meningitis, PilE has been shown to bind to sialylated N-glycans on the human endothelial cell receptor CD147 (also called EMMPRIN or Basigin) (Le Guennec et al., 2020) and the $\beta 2$ adrenergic receptor (Virion et al., 2019), two membrane proteins that form a heterotrimeric complex with cytoplasmic $\alpha$-actinin 4 (Maïssa et al., 2017).

The pathogenic Neisseria possess a set of core minor pilins, PilH (FimT), Pill (PilV), PilJ (PilW) PilK and PilX, that are encoded within a single gene cluster and prime pilus assembly (Winther-Larsen et al., 2005) plus "non-core" minor pilins PilV and ComP, which are encoded elsewhere on the genome. ComP shares the canonical T4P-pilin structure of the major pilin, PilE, with the N-terminal $\alpha$-helix and C-terminal globular domain (Berry et al., 2016). ComP is involved in natural transformation of exogenous DNA (Cehovin et al., 2013). PilV, which is highly conserved in N. meningitidis isolates (Cehovin et al., 2010), participates in adhesion and signaling in host cells (Winther-Larsen et al., 2001; Mikaty et al., 2009; Takahashi et al., 2012; Bernard et al., 2014; Virion et al., 2019). In N. meningitidis, PilV, like PilE, directly interacts with CD147 and the $\beta 2$-adrenergic receptor, suggesting that PilV colocalizes with PilE within the T4P filament (Bernard et al., 2014; Virion et al., 2019). However, another report concluded that PilV functions exclusively from within the periplasm, fine-tuning pilus surface display to regulate its interactions with host cells (Imhaus and Dumenil, 2014).

The T4P-receptor interaction represents a key step in $N$. meningitidis adhesion and colonization of endothelial cells in peripheral and brain vasculature and is thus an attractive target for preventive and therapeutic approaches to tackle meningococcal infection. Interfering 
with piliation prevents $N$. meningitidis colonization of human endothelial cells and vasculature

104 (Aubey et al., 2019) and improves sepsis outcome in a mouse model grafteded with human skin (Denis et al., 2019). Although both PilE and PilV are involved in adhesion, PilE exhibits considerable amino acid sequence variability in its exposed hypervariable region. This

107 variability contributes to Neisseria immune escape. In contrast PilV is highly conserved and 108 has been shown to be immunogenic in humans (Cehovin et al., 2011). Thus, PilV may prove to 109 be a more promising target than PilE for blocking endothelial cell adhesion. A molecular understanding of this minor pilin with respect to its structure, localization within the pilus and interactions with host receptors will be valuable in assessing its potential as a therapeutic target.

112 Here we report the atomic structure of PilV and super-resolution microscopy images showing 113 that it is incorporated throughout the $N$. meningitidis T4P. We identify residues involved in 114 adhesion to host cells and map these onto the PilV structure, modeled within the cryo-electron microscopy-derived pilus filament structure. Finally, we show that anti-PilV antibodies inhibit meningococcal adhesion in vivo. These data provide insights into PilV-mediated adhesion and

117 suggest that blocking its adherence functions may inhibit N. meningitidis vascular colonization 118 and pathogenesis. 
RESULTS

121

122

123

124

\section{PilE and PilV make distinct contributions to T4P-dependent meningococcal adhesion}

Both PilE and PilV have been shown to directly interact with host cell receptors (Bernard et al., 2014; Virion et al., 2019). To investigate their specific roles in meningococcal adhesion to human cells we compared adhesion for wild-type (WT) N. meningitidis strain 2C4.3 with that of derivative strains lacking either PilE or PilV on three endothelial cell types (Fig. 1A): primary human dermal microvascular endothelial cells (HDMEC) that are thought to have conserved their in vivo phenotype (Aird, 2012); the human cerebral microvascular endothelial cell line D3 (hCMEC/D3) that possesses characteristics of brain endothelial cells; and EA.Hy926 cells, obtained by fusion of human umbilical vein endothelial cells (HUVECs) with the human epithelial carcinoma cell line A549. While both $\Delta p i l E(\Delta E)$ and $\Delta p i l V(\Delta V)$ mutants showed substantial adhesion defects compared to WT bacteria, the defect of the $\Delta E$ mutant is more pronounced on primary human dermal microvascular endothelial cells (HDMECs) compared to the $\Delta V$ mutant (2.4 fold decrease between means of $\Delta V$ vs. $\Delta E$ ). PilV complementation of the $\Delta$ pilV strain $(\Delta V+V)$ partially restored adhesion to endothelial cells.

To assess the adhesive role of PilE in vivo we used a SCID mouse model grafted with human skin (Join-Lambert et al., 2013) to quantify bacterial adhesion on human vessels in vivo.

Grafted mice were infected intravenously with the WT, $\Delta E$ or $\Delta V$ mutant strains and bacterial loads in the graft and in the blood were quantified by counting colony forming units (CFUs) at different time points (Fig. 1B and supplemental Fig. 1A). Blood bacterial loads were identical between the three strains at 1 hour post-infection, indicating that equivalent inocula were administered for each bacterial strain (Supplemental Fig. 1A). Whereas more than $10^{8}$ CFUs were recovered 4 hours after infection from the graft infected with WT $N$. meningitidis, only 6 x $10^{3}$ CFUs were recovered from the grafts infected with PilE-defective meningococci, representing $\sim 0.0005 \%$ of the WT strain. The small number of $\Delta E$ bacteria are likely non- 
adherent cells present in the bloodstream of the graft microvasculature, as shown previously (Join-Lambert et al., 2013). The colonization efficiency of the PilV-defective strain was also impaired substantially at $\sim 0.08 \%$ of the WT strain, yet it was 170 -fold greater than that of the PilE-defective strain, suggesting an important role for PilV in human endothelial cell adhesion. We next assessed pilus levels in the $\Delta V$ strain to determine whether loss of piliation might explain its adhesion defect. T4P were mechanically sheared from WT, $\Delta V$ and $\Delta V+V$ strains by vortexing, separated from the cells by centrifugation, and concentrated using ammonium sulfate precipitation, producing a crudely purified pilus fraction that contains both major and minor pilins (Helaine et al., 2005; Winther-Larsen et al., 2001; Wolfgang et al., 1998). Pilus levels were reported as a piliation index, determined by quantifying the PilE content in sheared pili fraction normalized to the PilE in the bacteria fraction. As shown in Fig. 1C and Supplemental Fig. 1B, all three strains express equivalent amounts of PilE in the whole cell lysates, but PilE is reduced in the sheared fraction of the $\Delta V$ strain relative to WT, corresponding to a piliation index of $50 \%$. Thus, while deletion of PilV reduces pilus levels in the $\Delta V$ this reduction does not account for the pronounced adhesion defects observed for the PilV-defective strain in cell culture and in the mouse graft model. These results suggest that PilV is in fact the major adhesin on the N. meningitidis T4P.

\section{PilV-mediated adhesion is a conserved feature of $N$. meningitidis}

In contrast to PilE, which contains a surface exposed hypervariable loop providing extensive antigenic variation, PilV is conserved among clinical isolates (Cehovin et al., 2010). We examined 17,732 N. meningitidis genomes available in the PubMLST database, we identified 699 distinct PilV sequences having a mean sequence identity greater than $80 \%(86.6 \% \pm 5.9 \%$ SD) between every possible sequence pair, indicating high conservation (Supplemental Table S1). 


\section{N. meningitidis PilV is distributed throughout the T4P filament}

172 Both PilV and PilE were shown previously to bind to CD147 and the $\beta 2$-adrenergic (Bernard 173 et al., 2014; Virion et al., 2019), suggesting that PilV colocalizes with PilE in the pilus filament.

174 To investigate PilV localization and understand how it might serve as the main adhesin in vivo, 175 we tested for the presence of PilV in N. meningitidis T4P. Immunoblotting of ammonium sulfate-precipitated pili with anti-PilV antibody showed that PilV is present along with PilE (Supplemental Fig. 2A). This finding does not appear to be due to contamination of cellassociated proteins as the sheared fractions lacked both the cytosolic marker NADP-glutamatedeshydrogenase and the membrane marker, Rmp4 (Supplemental Fig. 2A). To further demonstrate that PilV is incorporated into the meningococcal T4P filament, we immunoprecipitated pili from a crude preparation using anti PilE and anti-PilV antibodies. PilV is present in the pilus fraction immunoprecipitated with anti-PilE antibody and, conversely, PilE is present in the pilus fraction immunoprecipitated with anti-PilV antibody, consistent with these proteins colocalizing within the pilus (Supplemental Fig. 2B, C).

To directly visualize PilV in the T4P filaments, we analyzed the spatial organization of both PilV and PilE at the molecular level $(\sim 20 \mathrm{~nm})$ using super-resolution microscopy technique based on single-molecule localization (dSTORM) (Maïssa et al., 2017; Denis et al., 2019). Anti-PilE antibody 20D9 binds in a continuous pattern to pili on WT cells grown in broth, whereas the pattern is punctate for adherent diplococci grown on endothelial cells, in which the pili are under stress (Biais et al., 2010; Brissac et al., 2012) (Fig. 2A, B). Surprisingly, PilV aslo shows a punctate and abundant distribution along the same filaments. As control, PilE but not PilV was detected on type IV filaments in the $\Delta p i l V$ mutant, confirming the specificity of the antibodies. Together, these data demonstrate that the minor pilin PilV is incorporated throughout the pilus filament consistent with its role as an adhesin. 


\section{Structure of $N$. meningitidis PilV}

197

To better understand the role of PilV in host cell adhesion, we solved the x-ray crystal structure of rPilV (residues 29-122, Fig. 3A, B) in two space groups, C2 (monoclinic) and P2 12121 (orthorhombic) at $1.41 \AA$ and $1.96 \AA$, respectively (Table 1). The structures are very similar, superimposing with a root mean square deviation (RMSD) of $0.5 \AA$ for main chain atoms. The higher resolution monoclinic structure is described here. The rPilV structure represents the pilin globular domain; the missing residues 1-28 correspond to the N-terminal half of an extended $\alpha$-helix, $\alpha 1$. rPilV possesses the canonical type IV pilin fold, with the C-terminal half of $\alpha 1$, $\alpha 1 \mathrm{C}$, packed against a 4-stranded antiparallel $\beta$-sheet (Fig. 3B). The $\alpha \beta$-loop that connects the top of $\alpha 1 \mathrm{C}$ to the bottom of strand $\beta 1$ is extended and has a central $3_{10}$ helix. On the other side of the $\beta$-sheet, a C-terminal loop curves under $\beta 4$ and links back to the $\beta$-sheet via a disulfide bond to the $\beta 2-\beta 3$ loop just before $\beta 3$ (Cys99 and Cys118). The disulfide bond delineates the D-region of the pilin. Short loops of 6 and 5 residues at the top of the globular domain connect strands $\beta 1-\beta 2$ and $\beta 3-\beta 4$, respectively, whereas the $\beta 2-\beta 3$ loop at the bottom of this domain is longer, with 12 amino acids.

Though, PilV has only limited sequence homology beyond $\alpha 1 \mathrm{~N}$ to the $N$. meningitidis major pilin PilE, and is substantially smaller, with 122 amino acids compared to PilE's 161 amino acids (Fig. 3A), PilV is similar in structure to PilE from both N. meningitidis and N. gonorrhoeae (Fig. 3D). The most significant differences are (i) the $\beta$-strands, which decrease in length from $\beta 1-\beta 4$ for PilV but become increasingly longer for PilE; the absence on PilV of the hypervariable $\beta$-hairpin loop insertion comprising much of the D-region of PilE; (ii) and the $\alpha 1 C$ structure, which is curved in PilE due to the presence of the helix-breaking Gly42, but straight in PilV, which has a histidine at this position (Fig. 3E). This straight $\alpha 1 \mathrm{C}$ is seen in other N. meningitidis minor pilins of known structure, the core minor pilin PilX and the 
competence-associated minor pilin ComP, despite PilX having a glycine at position 42 (Fig. 3E-G). However, all these pilins possess the conserved curvature-inducing Gly14 and Pro22. These conserved residues delineate a segment of $\alpha 1 \mathrm{~N}$ that is helical in the full-length pilin subunit but melted in the pilus filament, presumably to allow packing of $\alpha 1 \mathrm{~N}$ into the hydrophobic core of the filament (Kolappan et al., 2016; Wang et al., 2017). PilV is the smallest and simplest of the Neisseria pilins; each of the others have unique $\alpha \beta$-loop and/or D-region structural features that contribute to pilus functions and are predicted to be surface-exposed. The hypervariable $\beta$-hairpin contributes to immune escape for the Neisseria major pilins. The PilX D-region has a short "pigtail" $\alpha$-helix that is involved in pilus-mediated bacterial aggregation and adhesion (Helaine et al., 2007), and ComP has an extended loop that lies across the $\beta$-sheet and is implicated in DNA recognition (Berry et al., 2016).

\section{Model of minor pilin within pilus filament}

To understand how PilV might integrate into the pilus filament to influence its functions, the PilV structure was fit into the $N$. meningitidis pilus filament model, derived by cryoEM reconstruction (Kolappan et al., 2016), in place of one of the major pilins, PilE (Fig. 3H, Supplemental Fig. 3). The two $\alpha 1 \mathrm{Cs}$ were aligned at their $\mathrm{N}$ - and C-terminal ends, resulting in good superposition of the $\alpha \beta$-loop and $\beta$-sheet, including its loops, with no steric clashes. However, the PilV globular domain is smaller than that of PilE, with a shorter $\beta 3-\beta 4$ strandloop-strand and a more compact C-terminus, which leaves a gap between PilV and the adjacent PilE that exposes the melted region of $\alpha 1$ of a higher subunit (Fig. $3 \mathrm{H}, \mathrm{I}$ ). The structure of the exposed face of PilV, which lacks the hypervariable loop, and its chemistry (Fig. 3J) differs substantially from that of PilE (Fig. 3K). Thus, inserting PilV in place of PilE would change both the packing of the pilin subunits and the surface stereochemistry, which may impact the flexibility of the pilus and its interactions with host receptors. Importantly, the N. meningitidis 
T4P structure used in this model was built using only PilE as the building block (Kolappan et al., 2016). Our findings here show that PilV is distributed randomly throughout the pilus, which will have averaged out the electron density and likely limited the resolution of the reconstruction.

\section{Mutational scanning of pilV}

To identify the residues/regions of PilV responsible for interactions with host cell receptors, we used a two-step scanning mutagenesis. Mutations were introduced into the pilV gene inserted in the pKH37 plasmid (Ramsey et al., 2012), which was used to transform the PilV-deficient N. meningitidis $2 \mathrm{C} 4.3$ strain. Step 1: consecutive amino acid triplets throughout the globular domain were substituted with alanine triplets (or valine when the WT amino acid was alanine). Mutants expressing PilV variants within the pilus were screened for adhesion to hCMEC/D3 cells (Fig. 4A). Mutants that express PilV and incorporate this minor pilin into surface pili but showed poor adhesion indicate PilV defects related to adhesion rather than to protein stability, pilus biogenesis or integration into the pilus. Such mutants were selected for Step two (Fig. 4A and Supplemental Methods). Step 2: A panel of single amino-acid alanine substitutions were generated at sites associated with adhesion defects. These pilV mutants were expressed in the $\Delta V$ strain, as for the triplet mutants. The single amino acid mutants were tested for PilV localization within pili and then for adhesion to hCMEC/D3 cells (Fig. 4B). Of particular interest were the $\Delta V$ mutants that have WT levels of PilV in their sheared pilus fractions yet showed little or no adhesion (Fig. 4B and see Supplemental Methods): residues corresponding to these substitutions, 60-64 as well as L75, P91, G106, G107, A110 and G113 may be directly involved in host cell adhesion (see Supplemental Methods). To further confirm the roles of these amino acids in adhesion to endothelial cells, we introduced the corresponding alanine/valine changes into the pilV plasmid used to produce recombinant His-tagged PilV 
(rPilV-His, residues 29-122), and generated rPilV-His variants. As demonstrated previously (Bernard et al., 2014), addition of wild type rPilV-His reduces adhesion of meningococci to endothelial cells (hCMEC/D3) by $\sim 70 \%$, whereas a similar dose of recombinant minor pilin ComP (rComP-His) has no effect on binding (Supplemental Fig. 4A, B). The ability of these rPilV-His variant to inhibit $N$. meningitidis adhesion to hCMEC/D3 endothelial cells is shown in Fig. 4C and summarized in Supplemental Table S2. Among the rPilV-His variants that were produced at levels comparable to that of WT PilV-His, the rPilV-His-G113A variant inhibited adhesion at a level comparable to that of WT rPilV-His, ruling out this residue as a receptor binding candidate. rPilV-His-K62A showed a 2-fold inhibition of adhesion which was not statistically significant compared to buffer. rPilV-His-N63A, -K64A and -A110V showed only partial inhibition of adhesion and rPilV-His-Y60A, -D61A, -L75A and -G106A showed little to no inhibition. These results are consistent with the adhesion defects observed for the $N$. meningitidis PilV point mutants and implicate residues Y60-D61, N63-K64, L75, G106 and A110 in adhesion to endothelial cells.

These amino acids are highlighted on PilV in Fig. 4D-F. Residues Y60-K64 are centered on the 310 helix within the $\alpha \beta$-loop of PilV. The aromatic side chain of Tyr60 is buried within the globular domain and may be required to orient this loop and properly present the polar/charged side chains of Asp61, Asn63 and K64 on the PilV surface for binding to host cell receptors. Leu75 lies on strand $\beta 1$ and appears to stabilize the Y60-K64 loop, contacting the side chains of Tyr60, Leu65 and the aliphatic portion of the Lys62 side chain. The Y60-K64 loop protrudes from the globular domain and lies on the pilus surface and is adjacent to the PilE subunit in our filament model (Fig. 4E, F). Thus, this loop may influence the conformation of PilE and comprise part of a larger binding site that involves one or more PilE subunits. A graphical representation of the amino acid multiple sequence alignment shows Y60, D61 and K64 as conserved among the 699 distinct PilV sequences identified above, and K62 and N63 
are present in about 50\% of these sequences (Supplemental Table S1, Supplemental Fig. 4D), consistent with the importance of these residues in adhesion (Fig 4C). The three N. meningitidis genomes, 2C4.3, FAM18 and Z2491, which are commonly used in laboratory, have several amino acid differences but share the residues implicated in adhesion, Y60-K64, L75 and G106, while A110 is replaced by a serine in Z2491 PilV sequence (Fig. 4G). Consistent with these residues being involved in T4P-mediated adhesion, complementation of the $2 \mathrm{C} 4.3 \Delta V$ with pilV of FAM18 or Z2491 had no significant impact on adhesion to endothelial cells (Supplemental Fig. 4C).

Interestingly, no pilV mutants were identified that produced wild type levels of PilV that were not incorporated into the pilus. Such mutants might have indicated residues involved in PilV:PilE interactions within the pilus. However, these results are consistent with our knowledge of Type IV pilus structure, in which subunits are held together primarily by interactions among $\alpha 1 \mathrm{~N}$ residues: hydrophobic interactions as well as a salt bridge between Glu5 and the N-terminal amino group (Li et al., 2012; Kolappan et al., 2016; Wang et al., 2017). To test the role of key residues in $\alpha 1 \mathrm{~N}$ we substituted the conserved Phe1 and Glu5 with alanine. As predicted these changes do not impact PilV expression but PilV is poorly incorporated into the pilus (Supplementary Fig. 5). Pilus levels, as assessed by PilE in the crude pilus preparations, are not affected in these mutants.

\section{Anti-PilV antibodies inhibit $N$. meningitidis adhesion}

We next investigated the potential for PilV targeted antibodies to inhibit bacterial adhesion in vivo using the human skin-grafted mouse model (Join-Lambert et al., 2013). Anti-PilV polyclonal antibodies were produced in rabbits immunized with $\mathrm{rPilV}$ and monoclonal antibodies were produced in mice immunized with PilV peptides spanning the residues implicated in adhesion (peptide 58-66, 98-119) and the $\beta$-strand 2 (peptide 80-97). No 
antibodies were obtained in mice with the peptide spanning neither the Y60-K64 adhesive loop nor the 98-119 D-region. Only immunizations with peptide 80-97 (residues PDHFTLQADPNPTTNDGE of mature PilV) were successful, resulting in four monoclonal antibodies. Antibodies were injected in the tail vein of grafted animals two hours prior to 324 intravenous infection with WT N. meningitidis. Bacterial colonization of human skin grafts was 325 determined 4 hours after infection by counting CFUs (Fig. $4 \mathrm{H}$ ). Two different batches of rabbit 326 polyclonal antibodies raised against rPilV reduced vascular colonization by 9.8-fold and 3.4327 fold respectively, relative to the unimmunized controls. Two different mixtures of monoclonal 328 antibodies raised against peptide 80-97 also significantly inhibited vascular colonization 329 (6E9+17C7 by 2.4 -fold; $16 \mathrm{C} 1+12 \mathrm{D} 4$ by 2.8 -fold), while rabbit and mouse isotype control 330 antibodies had no effect. The reduced colonization in the presence of these antibodies is not due 331 to a bactericidal effect of the antibodies since counts of CFU in the blood of the grafted mice were not statistically different between the control and antibody groups 4 hours after infection

333 (Supplemental Fig. 6). These results demonstrate that anti-PilV antibodies can efficiently block N. meningitidis T4P-dependant adhesion to human host-cell receptors. 


\section{DISCUSSION}

337 Here we show that the conserved non-core minor pilin PilV is involved in adhesion to host endothelial cells by virtue of its incorporation throughout the pilus filament. PilV has a canonical type IV pilin fold but lacks the surface adornments seen in PilE (the hypervariable $\beta$ hairpin), PilX (the $\alpha$-helix “pigtail”) and ComP (the DNA-binding strand). When modeled into the pilus filament in place of PilE a gap is created that may expose additional binding sites and influence filament flexibility.

To our knowledge this is the first demonstration of a minor pilin being abundantly distributed throughout the pilus, and indeed calls into question its designation as a minor pilin. Core minor pilins are encoded within a single gene cluster and have been shown to interact with each other and with the major pilin (Nguyen et al., 2015). These low abundance pilins share the canonical type IV pilin fold and are thought to form a cluster that initiates pilus assembly and caps the filament. In contrast to the core minor pilins, both PilV and ComP are encoded in separate sites on the N. meningitidis genome. They share the pilin fold but have specific roles in the bacterial life cycle, in adhesion and DNA uptake, respectively. The reduction in pili in the $\Delta$ pilV mutant suggests that PilV may have an additional non-essential role in pilus assembly or stability. The localization of PilV, and presumably ComP, further distinguish them from the putative tip-associated core minor pilins. These non-core minor pilins are unique to the pathogenic Neisseria, perhaps because their major pilin undergoes antigenic variability. domain relative to that of the major pilin PilE. The critical Y60-K64 adhesion loop identified by iterative alanine scanning mutagenesis appears to be located in close apposition to PilE, in a shallow well beneath the protruding, hypervariable $\beta$-hairpin of PilE. The Y60-K64 loop is conserved among $N$. meningitidis strains, and thus provides a recessed receptor binding site, distributed abundantly along the length of the pilus. We show here that PilV mediates adhesion 
to host cells in vitro and in vivo. Yet rather than simply providing a filamentous scaffold for the primary adhesin PilV, PilE is itself adhesive, as the piliated $\Delta V$ mutant retains some adhesion capability, whereas a non-piliated $\Delta E$ mutant is non-adherent. Our data are consistent with previous observations that both PilE and PilV interact with host cell receptors and are required for efficient adhesion (Bernard et al., 2014; Virion et al., 2019; Le Guennec et al., 2020). Our findings help to explain how N. meningitidis can alter the amino acid sequence of its major pilin from one generation to the next to evade the host immune response while maintaining its ability to adhere to host endothelial cells. The hypervariable PilE $\beta$-hairpin is prominently displayed on the pilus surface and may elicit neutralizing antibodies, but these would be rendered ineffective due to antigenic variation, whereas the recessed and conserved PilV maintains adhesion capability for the pilus.

The recessed nature of the Y60-64 loop in the context of the intact pilus makes it an even less likely epitope for recognition by human antibodies. Nonetheless, monoclonal antibodies targeting peptide 80-97 and rabbit sera raised against rPilV inhibit vascular colonization in vivo, demonstrating the viability of PilV in eliciting a protective immune response. Consistent with these findings, convalescent sera from patients with meningococcal disease recognized PilV in an ELISA assay (Cehovin et al., 2011), although their impact on bacterial adhesion was not tested. The ability of anti-PilV antibodies to inhibit vascular colonization by $N$. meningitidis in a human skin graft is a key finding of this study. PilV may be the Achilles heel of the $N$. meningitidis $\mathrm{T} 4 \mathrm{P}$, providing a conserved and critical target to block adhesion and treat meningococcal infections, and a protective antigen for meningococcal subunit vaccines. A finding that may also be relevant for $N$. gonorrhoeae, which also possesses PilV. 


\section{Bacterial strains and endothelial cells}

385

Neisseria meningitidis 2C4.3 strain, (formerly clone 12) that is a piliated encapsulated OpaOpc- variant of the serogroup C meningococcal clinical isolate NEM8013 and its isogenic nonpiliated PilE-defective mutant $(\Delta E)$, the PilV-defective mutant $(\Delta V)$ and the PilVcomplemented strain $\left(\Delta V+V_{2 C} 4.3\right)$ were described before (Bernard et al., 2014). The pilV sequence of strains FAM18 (Dyer et al., 1987) or Z2491 (Achtman et al., 1988) were extracted from genomic DNA by PCR and pilV genes were inserted by Gibson assembly in plasmid pKH37 between the $N$. meningitidis genes $l c t P$ and $a s p C$ and downstream of the lacP promoter (Ramsey et al., 2012). Bacterial strains were stored frozen at $-80^{\circ} \mathrm{C}$ and routinely grown at $37^{\circ}$ $\mathrm{C}$ in a moist atmosphere with $5 \% \mathrm{CO}_{2}$ on gonococcal base (GC) agar plates (Difco) containing Kellogg's supplements. Antibiotics were added as indicated. Strains, plasmids and primers are listed in Supplemental table S3.

Human Cerebral Microvascular Endothelial Cells (hCMEC/D3) are a fully differentiated brain endothelial cell line derived from human brain capillaries that recapitulate the major phenotypic features of the blood-brain barrier (Weksler et al., 2005). hCMEC/D3 were grown onto Cultrex rat collagen type I-coated dishes (R\&D) in Endothelial Cell Basal Medium-2 (Lonza) supplemented with 5\% of fetal calf serum (FCS), $1.4 \mu \mathrm{M}$ hydrocortisone (Lonza), $5 \mu \mathrm{g} / \mathrm{mL}$ ascorbic acid (Lonza), $1 \mathrm{ng} / \mathrm{mL}$ b-FGF (Lonza), at $37{ }^{\circ} \mathrm{C}$ in $5 \% \mathrm{CO}_{2}$. Human Dermal Microvascular Endothelial Cells (HDMEC) were purchased from Promocell and grown onto Cultrex rat collagen type I-coated dishes (R\&D) in Endothelial Cell Growth Medium MV (Promocell) supplemented with Endothelial Cell Growth Medium Supplement Mix (Promocell), at $37{ }^{\circ} \mathrm{C}$ under 5\% CO2. EA.Hy926 (ATCC CRL-2922) are endothelial cells established by fusing primary human umbilical vein cells with a thioguanine-resistant clone of A549. Cells are grown in Dulbecco's Modified Eagle's Medium (DMEM) supplemented with 
$10 \% \mathrm{FCS}$, at $37{ }^{\circ} \mathrm{C}$ under $5 \% \mathrm{CO}_{2}$.

\section{Endothelial cell infection and adhesion assays}

On the day of infection, a suspension of bacteria from an overnight culture on GCB agar plate was adjusted to $\mathrm{OD}_{600} 0.05$ and incubated for 2 hours at $37{ }^{\circ} \mathrm{C}$ in pre-warmed cell culture medium (according to cell type). Cells were infected with bacteria at a multiplicity of infection (MOI) of 100 bacteria per cell for $30 \mathrm{~min}$, washed six times to remove unbound bacteria and colony forming units (CFU) were counted by plating serial dilutions on GCB agar plates. When required, cells were treated with recombinant His--PilV at indicated concentrations for $30 \mathrm{~min}$ before infection.

\section{Infection of human skin grafted mice}

Six to 8-week old CB17/Icr-Prkdc scid (Severe Combined Immunodeficiency: SCID) female mice were obtained from Janvier Labs (Saint-Berthevin, France). Mice were grafted with normal human skin as previously described (Join-Lambert et al., 2013). Briefly, full thickness human skin was grafted onto the back of SCID mice by surgical stitching. Grafted mice were randomized into control and treated groups. When required, anti-PilV antibodies or buffer (physiological saline) were administered intravenously 2 hours prior infection at a total dose of $100 \mu \mathrm{g}$ for polyclonal anti-PilV or $50 \mu \mathrm{g}$ each of two monoclonal anti-PilV preparations. $N$. meningitidis strains were grown overnight on GCB agar plates without iron (Kellogg supplement II) and supplemented with deferoxamine (Desferal, Novartis) at $37^{\circ} \mathrm{C}$. On the day of infection, bacteria were harvested and grown under agitation in RPMI medium with 1\% bovine serum albumin (BSA) and $0.06 \mu \mathrm{M}$ deferoxamine to exponential phase. Bacteria were then resuspended in physiological saline and mice were infected intravenously with $N$. meningitidis 2C4.3 WT strain or the mutant strains defective for PilE or PilV ( $\Delta E$ and $\Delta V)(5 \mathrm{x}$ 
$10^{5}$ or $5 \times 10^{6}$ bacteria). Bacteremia was assessed at 1 hour and 4 hours by collecting a blood sample by tail vein puncture. Mice were sacrificed 4 hours after infection and human skin grafts were removed sterilely. The grafts were crushed and homogenized with Lysing Matrix M tubes using FastPrep (MP Biomedicals) with 2 cycles of $15 \mathrm{~s}$ at speed $6 \mathrm{~m} / \mathrm{s}$. Bacterial counts were determined by plating serial dilution of the samples onto GC agar plates.

\section{Crude pilus purification}

Ammonium sulfate precipitation. $N$. meningitidis strains were grown overnight on GCB agar plates, scraped off of the plates and resuspended in $2 \mathrm{~mL}$ of $20 \mathrm{mM}$ ethanolamine, $\mathrm{pH} 10.5$ supplemented with $1 \mathrm{mM}$ dithiothreitol (DTT) at $4{ }^{\circ} \mathrm{C}$. To shear pili off, the bacteria were vortexed vigorously 3 times for 1 min bursts, returning them to ice for 1 min between each burst. Bacterial cells were removed from the pilus suspension by two successive centrifugations at $10000 \mathrm{x} \mathrm{g}$ for $20 \mathrm{~min}$ at $4{ }^{\circ} \mathrm{C}$. Cell pellets were resuspended in lysis buffer $(50 \mathrm{mM}$ Tris $\mathrm{pH}$ 7.5, $25 \mathrm{mM}$ HEPES, $2 \mathrm{mM}$ EDTA, 1\% (w/v) SDS - bacterial fraction). The supernatant containing the pili was collected and supplemented with saturated ammonium sulfate in $20 \mathrm{mM}$ ethanolamine, $\mathrm{pH} 10.5$ at a final concentration of $0.15 \mathrm{M}$, before overnight agitation at $4{ }^{\circ} \mathrm{C}$. Aggregated pili were pelleted by centrifugation at $17000 \mathrm{x}$ g for $20 \mathrm{~min}$ at $4{ }^{\circ} \mathrm{C}$ and resuspended overnight at $4{ }^{\circ} \mathrm{C}$, in $400 \mu \mathrm{l}$ of $20 \mathrm{mM}$ ethanolamine, $\mathrm{pH} 10.5$. The pili solution was centrifugated at $10000 \mathrm{xg}$ for $20 \mathrm{~min}$ at $4{ }^{\circ} \mathrm{C}$ to remove residual cell debris and the supernatant was concentrated 10-fold using an Amicon 10 kDa MWCO membrane (Merck Millipore).

\section{Colocalization of PilV and PilE by immunoprecipitation}

Bacteria grown on GC agar plates were adjusted to $\mathrm{OD}_{600}=0.2$ in prewarmed liquid GCB medium then incubated at $37{ }^{\circ} \mathrm{C}$ under $5 \% \mathrm{CO}_{2}$ during $1 \mathrm{~h}$. Bacterial suspension was then vortexed, passed through a $26 \mathrm{G}$ needle and centrifugated at $1500 \mathrm{~g}$ for $15 \mathrm{~min}$. The cleared 
supernatants were used for immunoprecipitation with specific antibodies against PilE (5C5 $\mathrm{mAb}$ ) or PilV (rabbit serum Acfp) coated on protein $\mathrm{G}$ magnetic beads (Pierce Thermo Scientific). Precipitated proteins were separated on SDS-PAGE gels and transferred to nitrocellulose (GE Healthcare). After blocking for $1 \mathrm{~h}$ in PBS - 1\% BSA - 0.05\% Tween-20, filters were probed overnight with specific antibodies against PilE (rabbit polyclonal antibodies \#9410), PilV (Acfp), anti-NADP-glutamate-deshydrogenase (NADPGH, cytosolic marker) and anti-Rmp4 (membrane marker) and revealed in ECL (Thermo Scientific), after probing with peroxidase-coupled secondary antibodies (GE Healthcare).

\section{Immunoblotting and piliation index calculations}

Bacteria grown overnight were scraped from GC plates and resuspended in PBS and Laemmli buffer for whole bacterial lysate samples. Laemmli buffer was added to pilus preparations and bacterial fractions (see Crude pilus purification). Samples were analyzed for the presence of PilV, PilE, NADP-glutamate-dehydrogenase or Rmp4 by SDS-PAGE and immunoblotting. The piliation index was determined by quantifying PilE in sheared pilus fraction based on densitometry of immunoblots bands and normalizing this value to the PilE content in the corresponding bacterial fraction (ImageJ Software).

\section{PilV sequence comparison}

All sequences correspond to the query pilV (NEIS0487) in the MRF Meningococcus Genome Library (PubMLST) database (864 non-redundant nucleotide sequences on the $14^{\text {th }}$ January 2019). DNA sequences were translated into protein sequences using EMBOSS Transeq, https://www.ebi.ac.uk/Tools/st/emboss transeq/). The redundancy of the corresponding protein sequence dataset was reduced using CD-HIT v4.7 (Fu et al., 2012) with a 100\% identity threshold yielding 699 clusters. The longest representative sequence of each cluster was aligned 
with Clustal Omega v1.2.4 (Sievers and Higgins, 2014) using the default parameters. We then used the SIAS (Sequence Identity And Similarity) server (http://imed.med.ucm.es/Tools/sias.html) to calculate pairwise sequence identity and similarity from the multiple sequence alignment with default parameters for similarity, amino acid grouping and length of multiple sequence alignment for the denominator (Supplemental Table S1). A graphical representation of the amino acid multiple sequence alignment is shown as a sequence logo (Crooks et al., 2004) generated by using Weblogo 3.7.4.

\section{Stochastic optical reconstruction microscopy (dSTORM)}

Bacteria were grown in Endothelial Cell Growth medium MV (Promocell), spread in $22 \mathrm{~mm}$ high-precision cover glasses with thickness of $170 \mu \mathrm{m}$, (No. 1.5H, Marienfield) with cytospin and fixed in 4\% paraformaldehyde for 10 min before immunolabelling. HDMEC cells were seeded on the high-precision cover glasses (No. 1.5H, Marienfield), infected with meningococci for $90 \mathrm{~min}$, and fixed in 4\% paraformaldehyde for $10 \mathrm{~min}$. Bacteria or endothelial cells were blocked with 3\% BSA-PBS for 10 min, labelled with the monoclonal anti-PilE antibody 20D9 and the polyclonal anti-PilV antibody $6488\left(1: 100\right.$ in $2 \%$ BSA-PBS) $4{ }^{\circ} \mathrm{C}$ overnight, then incubated with goat anti-mouse Alexa Fluor $647 \mathrm{~F}\left(\mathrm{ab}^{\prime}\right) 2$ secondary antibody fragments and goat anti-rabbit Alexa Fluor 555 F(ab')2 secondary antibody fragments (Life Technologies; A21237) (1:500). Marienfield cavity slides were poured with blinking reagent (Smart Kit buffer optimized for dSTORM, Abbelight) and the cover glasses were sealed with a Twinsil 22 siliconglue (Rotec) as described previously (Maïssa et al., 2017). Images were acquired on a Leica SR GSD system, with a x 160 oil (NA 1.43) objective. Fluorescence images were collected using an EMCCD camera (iXon Andor technology), providing an effective pixel size of $100 \mathrm{~nm}$ and processed with the LAS X Software (Leica). Approximately 50,000 frames were recorded for each acquisition with $10 \mathrm{~ms}$ exposure time, EM gain of 300 and the number of photons per 
pixels set to $75.2 \mathrm{D}$ reconstructions were obtained from stacks (16 planes) with $50 \mathrm{~nm}$ axial step size, using the LAS X Software (Leica) or ImageJ.

\section{PilV expression, crystallization and structure determination}

Methods for PilV expression for crystallization and that of His-tagged PilV and His-tagged

ComP for competition assay are described in supplemental methods.

Crystallization and structure determination. Initial crystallization conditions for PilV were identified using the High-Throughput Crystallization Screening Center at the HauptmanWoodward Medical Research Institute (Luft). Conditions were optimized in house using the hanging drop vapour diffusion method. Crystals were grown in $100 \mathrm{mM}$ sodium acetate $\mathrm{pH}$ 5, $100 \mathrm{mM}$ ammonium chloride, $28 \%$ (w/v) PEG 8000. The crystals were cryo-cooled in mother liquor containing $30 \% \mathrm{v} / \mathrm{v}$ glycerol as cryoprotectant. Crystals were screened at the Stanford Synchrotron Radiation Lightsource (SSRL) Beamline 7-1 and x-ray diffraction data were collected at Beamlines 7-1 and 12-1 using Blu-Ice (Gonzalez) (Table 1). PilV datasets were processed, integrated, and scaled using XDS and the CCP4 programs iMosflm, POINTLESS, AIMLESS, \& TRUNCATE. PilV crystals belonging to 2 different space groups, monoclinic C2 and orthorhombic P2 $2{ }_{1} 21$, were obtained in similar crystallization conditions. Data were collected for the orthorhombic crystal form at wavelengths corresponding to inflection point and high energy remote and the PilV structure was solved by the 2-wavelength anomalous diffraction method (Table 1). The automated structure solution program SOLVE located two selenium sites and initial phases were calculated. Density modification was performed using RESOLVE resulting in an interpretable electron density map. A Matthews coefficient of 2.1 was calculated for the orthorhombic crystal, indicating a single molecule in the asymmetric unit with $42.4 \%$ solvent content. An initial model for PilV was built in COOT. The model was refined and water oxygens were located using the PHENIX suite. R-values for the refined model 
are $\mathrm{R}_{\mathrm{work}}=0.185$ and $\mathrm{R}_{\mathrm{free}}=0.255$ with a resolution of $1.96 \AA$. Data were collected to $1.4 \AA$ for one of the monoclinic PilV crystals. The Matthews coefficient is 1.9 for this crystal, giving 1 molecule per asymmetric unit and a solvent content of $33.8 \%$. The monoclinic PilV structure was solved by molecular replacement method using the lower resolution orthorhombic PilV structure as a model. PHASER gave a solution with high Z-scores $(\mathrm{RFZ}=7.6 \&$ and TFZ $=$ 24.3). This model was further improved using the crystallographic macromolecular model building program ARP/wARP. Several cycles of fitting and refinement were performed in COOT and PHENIX, respectively. Water oxygens were located and final PilV model was anisotropically refined with hydrogen atoms. Both monoclinic and orthorhombic crystal structures were validated using MOLPROBITY. The resolution of the monoclinic PilV structure is $1.41 \AA$, with an $\mathrm{R}_{\text {work }}$ of 0.172 and an $\mathrm{R}_{\text {free }}$ of 0.206 . Data collection and model statistics are given in Table 1.

\section{Scanning mutagenesis}

The mutations were introduced by PCR mutagenesis (primers are listed in Supplemental Table S3) into the pilV gene inserted in pKH37 plasmid. Plasmids were used to transform the PilVdeficient $N$. meningitidis $2 \mathrm{C} 4.3$ strain $(\Delta V)$. To examine the impact of the alanine substitutions, complemented $\Delta V$ mutants were first tested for their ability to assemble $\mathrm{T} 4 \mathrm{P}$ and to incorporate PilV into the pili by shearing the pili of the bacterial surface, concentrating them using ammonium sulfate and assessing PilE and PilV levels by SDS-PAGE and immunoblotting. Next, the piliated alanine substituted mutants were tested for their ability to adhere to hCMEC/D3 endothelial cells. Mutations in PilV sequence affecting PilV incorporation into pilus filament or T4P stability were excluded of our study.

\section{Generation of anti-PilV antibodies}


Anti-PilV polyclonal antibodies were produced in two rabbits immunized with rPilV (Proteogenix, France). Anti-PilV monoclonal antibodies were produced in mice against three different PilV peptides (peptide 58-66, 80-97 and 98-119). Hybridomas were selected for their production of antibodies that inhibited bacterial adhesion to human endothelial cells. Selected hybridomas were amplified and antibodies were purified. No antibodies for peptides 58-66 and 98-119 were obtained, either because they are not immunogenic or hybridomas were unstable. Two different antibody-producing companies were involved (Proteogenix, France; Biotem, France). Peptide 80-97 allowed for hybridoma selection and purification of antibodies (Biotem, France). Isotype control antibodies were produced and purified by Biotem, France.

\section{Statistical analysis}

Statistical analyses were performed with GraphPad Prism. Multiple comparison analyses were assessed with a one-way ANOVA or a one-way Brown-Forsythe and Welch ANOVA test depending on the variance analysis and data were expressed as mean $\pm 95 \% \mathrm{CI}$ (relevant $p$ values were reported in the figures). In case of failed normality test or positive Bartlett's test and important differences in mean and SD, a Kruskal-Wallis test was used and data were expressed as median \pm interquartile range (corrected $p$ values were reported in the figures). The $\mathrm{H} 0$ hypothesis was rejected for a significance level of $\mathrm{p} \leq 0.05$. Multiple comparison reports and descriptive statistics are available in Supplemental Statistics.

\section{Ethics Statements}

The animal experimental procedures described in this work conform to the European ethical regulations (Directive 2010/63/EU). The project was approved by Comité d'Ethique en matière d'Expérimentation Animale Paris Descartes and the Ministère de 1'Eduction Nationale de l'Enseignement Supérieur et de la Recherche (Project Number APAFIS\#16345- 
5832018012515596498 v5). Human skin tissues were obtained from surgical wastes from patients

584 undergoing plastic surgery (Groupe Hospitalier Paris Saint-Joseph). In accordance with the 585 French legislation, the study was declared to the Comité d'Ethique de la Recherche Paris 586 Descartes and patients were informed of the research purpose and oral consent was recorded.

587

588 ACKNOWLEDGEMENTS

589 This work was supported by research grants ANR-15-CE15-0002-01 (to MC), Fondation pour 590 la Recherche Médicale (XN and EC), ANR-14-IFEC-0006-01 (to SB and XN), INSERM and 591 Université de Paris (to MC, SB, XN) and by a Natural Sciences and Engineering Research 592 Council of Canada grant (to LC).

593 


\section{FIGURE LEGEND}

Figure 1. Differential adhesion of PilE and PilV defective strains of $N$. meningitidis. (A) HDMECs, hCMEC/D3 and EA.Hy926 human endothelial cells were incubated 30 min with meningococci (wild type: WT; $\Delta p i l E: \Delta E ; \Delta p i l V: \Delta V ; \Delta p i l V$ complemented strain: Cp). Following infection, unbound bacteria were removed and adherent bacteria were quantified by plating serial dilution on GCB agar plates and counting CFUs after overnight growth. Adhesion is expressed as the mean $\pm 95 \%$ CI (Confidence Interval) of CFUs normalized to the control infection (WT). Data were analyzed using Brown-Forsythe and Welch ANOVA. (B) Humanskin grafted SCID mice were infected intravenously with $5 \times 10^{6}$ N. meningitidis (WT; $\Delta E ; \Delta V$ ). Graft bacterial loads were quantified at 4 hours after infection by serial dilutions on GCB agar plates. Two independent experiments performed with a skin batch from a different donor. Each dot represents a single mouse; data are expressed as $\log 10$ of the mean $\pm 95 \%$ CI of CFU/g (B). Data were analyzed using Bonferroni's multiple comparisons. (C) Piliation index. Pili of WT, $\Delta V$ and $\Delta$ pilV complemented strain $(\Delta V+V)$ were sheared from the bacterial cells by vortexing and then precipitated with ammonium sulfate (AS). To determine the piliation index, the sheared pilus fraction and the bacteria fraction were analyzed by SDS-PAGE and immunoblotting with anti-PilE antibodies. Piliation indices were normalized to that of the WT. Data are expressed as the mean $\pm 95 \% \mathrm{CI}$ and were analyzed using Brown-Forsythe and Welch ANOVA.

Figure 2. PilV is distributed throughout the $N$. meningitidis type IV pilus. $(\mathrm{A}, \mathrm{B}, \mathrm{C})$ dSTORM images of $N$. meningitidis grown in liquid broth (wild-type or $\Delta p i l V$ ) or attached to endothelial cells (wild-type). Images were acquired on a Leica SR GSD 3D system. Fifty thousand frames were recorded and reconstructed using LAS X Software (scale bar, $2 \mu \mathrm{m}$ ). 
Figure 3. X-ray crystal structure of $N$. meningitidis PilV and model of PilV within $N$. meningitidis type IV pilus. (A) Sequence alignment of the $N$. meningitidis $(\mathrm{Nm})$ minor pilin PilV (NCBI WP_002244869) and the major pilin PilE (WP_014573675). Identical residues are highlighted orange and conserved residues in yellow. Helix-breaking Gly and Pro in $\alpha 1$ are highlighted in cyan and are boxed in black, as are Cys. Residues implicated in host-cell adhesion are boxed in blue. Secondary structures are indicated. The hypervariable region of PilE is underlined. (B) Crystal structure of $N$. meningitidis recombinant PilV, residues 29-122. The $\alpha \beta$-loop between $\alpha 1$ and the $\beta$-sheet is colored green and the D-region, delineated by the disulfide-bonded cysteines is magenta. Cysteines are shown as yellow sticks. The histidine at position 42 is colored cyan. (C) N. meningitidis major pilin PilE (residues 29-161, PDB 5JW8). (D) N. gonorrhoeae ( $\mathrm{Ng}$ ) major pilin PilE (full-length, residues 1-158, 2HI2). (E) Sequence alignment of $\alpha 1$ for N. meningitidis pilins and N. gonorrhoeae PilE, colored as in A (Nm PilX, CWT82783; Nm ComP, WP_002218144; Ng PilE, P02974). (F) $N$. meningitidis minor pilin PilX (residues 28-147, 2OPE). (G) N. meningitidis minor pilin ComP (residues 29-118, 5HZ7). Residues at positions 14 and 22 (N. gonorrhoeae PilE) and 42 (all pilins) are colored cyan. (H) The globular domain of a PilE subunit in the N. meningitidis T4P reconstruction (5KUA) was replaced with the rPilV structure (blue) by superimposing the $\mathrm{N}$ - and $\mathrm{C}$-terminal ends of $\alpha 1 \mathrm{C}$, leaving only $\alpha 1 \mathrm{~N}$ of PilE (red). All other PilE subunits are colored grey, with $\alpha 1$ (residues 155) shown in yellow and $\alpha 1 \mathrm{~N}$ residues Gly14 and Pro22 in cyan. The model is shown in cartoon (left) and space filling representations (middle and right). The filament has been rotated about its long axis in the right panel to show how the narrower PilV globular domain exposes $\alpha 1 \mathrm{~N}$ of a higher PilE subunit (dashed oval). (I) For comparison, the N. meningitidis T4P reconstruction is shown with a single PilE subunit colored red. (J, K) Electrostatic surface representation of PilV (J) and PilE (K), shown in approximately the same orientation as in (H) and (I). 

defective for adhesion. Volcano plots, adhesion of $N$. meningitidis $\Delta$ pilV strain expressing PilV variants. Data are shown for mutants in which pili are produced at approximately WT levels and PilV is detected in the pilus preparation. Adhesion is expressed as $\log 2$ of Fold Change between mutated and wild type PilV $(\log 2(\mathrm{FC}))$ and statistical significance is expressed as $\log 2(q$ value $)(-\log 2(q))$. Red dot represents mutant defective for adhesion. (A) Mutants selected during the first step of mutagenesis with triplet Ala/Val substitutions; (B) mutants selected during the second step of mutagenesis looking at single Ala/Val substitutions at sites of adhesion defects (see Supplemental Methods). (C) The ability of WT rPilV and rPilV variants to competitively inhibit $N$. meningitidis adhesion to hCMEC/D3 cells was assessed. Endothelial cells were treated with $50 \mu \mathrm{g} / \mathrm{mL}$ of recombinant proteins for 30 min prior to infection with $N$. meningitidis WT strain for 30 minutes. The number of adherent cell-associated bacteria was determined by counting CFUs. Data shown are averaged values for two independent experiments. Adhesion is expressed as $\log 2($ mean $) \pm 95 \% \mathrm{CI}$ of fold inhibition, normalized on bacterial adhesion of cells treated with buffer only. Statistical analyses were performed against buffer using the Kruskal-Wallis test. (D-F) N. meningitidis T4P structure with one PilE subunit replaces with PilV as for Fig. 3. (D) Cartoon representation of the pilus with PilV shown in blue, PilE in grey with $\alpha 1$ colored yellow. Residues implicated in host cell binding are shown in stick representation with carbon atoms in green, nitrogens blue and oxygens in red. The YDKNK loop is the Y60-K64 loop. (E, F) Cartoon and space-filling representations of the pilus model rotated about its axis to show how the Y60-K64 loop contacts the neighboring PilE and may form part of a larger receptor binding site. The hypervariable loop, which is part of the protruding $\beta$-hairpin of PilE is colored magenta in (F). (G) Alignment of PilV protein sequences from N. meningitidis 2C4.3, FAM18, Z2491 strains and that of the PubMLST consensus sequence (see Supplemental Fig. 4). Conserved residues are highlighted in red and non- 
conserved residues are highlighted in blue. Residues implicated in host-cell adhesion are boxed

670 in red. (H) Graft bacterial loads of human-skin grafted SCID mice injected intravenously with

671 buffer (control), $100 \mu \mathrm{g}$ of anti-PilV rabbit polyclonal IgG antibodies (\#1, \#2), $100 \mu \mathrm{g}$ of anti672 PilV peptide mouse monoclonal IgG antibodies (raised against the 673 P80DHFTLQADPNPTTNDGE97 peptide: 6E9+17C7, 16C1+12D4) or their respective isotype 674 control antibodies, followed by infection with $5 \times 10^{5} \mathrm{CFU}$ of WT meningococci. Graft bacterial 675 loads were determined 4 hours after infection and normalized to that of the control (buffer only). 676 Values shown are the averages of two independent experiments

677 performed with a skin patch from different donors. Each dot represents a single mouse; data 678 are expressed as the mean $\pm 95 \% \mathrm{CI}$ of CFU/g. Statistical analyses were performed against the 679 control with Brown-Forsythe and Welch ANOVA.

680 


\begin{tabular}{|c|c|c|c|}
\hline \multirow{2}{*}{$\begin{array}{l}\text { Data collection } \\
\text { SSRL beamline }\end{array}$} & \multicolumn{2}{|c|}{ PilV (orthorhombic) } & \multirow{2}{*}{$\begin{array}{l}\text { PilV (monoclinic) } \\
\text { SSRL 12-2 }\end{array}$} \\
\hline & SSRL 7-1 & SSRL 7-1 & \\
\hline Wavelength $(\AA)$ & 0.976 & 0.979 & 0.969 \\
\hline Space group & $\mathrm{P} 22_{1} 2_{1}$ & $\mathrm{P} 22_{1} 2_{1}$ & $\mathrm{C} 2$ \\
\hline \multicolumn{4}{|l|}{ Unit-cell parameters } \\
\hline $\mathrm{a}, \mathrm{b}, \mathrm{c}(\AA)$ & $21.8,39.3,109.6$ & $21.8,39.3,109.6$ & $46.3,21.8,83.8$ \\
\hline$\alpha, \beta, \gamma\left(^{\circ}\right)$ & $90,90,90$ & $90,90,90$ & 90104,90 \\
\hline Resolution $(\AA)$ & 1.96 & $1.96(2.07-1.96)$ & $1.41(1.43-1.41)$ \\
\hline Solvent content $(\%)$ & 42.4 & 42.4 & 33.8 \\
\hline Molecules/AU ${ }^{1}$ & 1 & 1 & 1 \\
\hline Total no. of reflections ${ }^{2}$ & $53268(2538)$ & $52638(6230)$ & $91105(4625)$ \\
\hline Unique reflections & $7263(442)$ & $7182(947)$ & $15424(772)$ \\
\hline Multiplicity & $7.3(5.7)$ & $7.3(6.6)$ & $5.9(6.0)$ \\
\hline Completeness (\%) & $98.7(89.2)$ & $98.8(94.6)$ & $95.9(97.0)$ \\
\hline$\langle\mathrm{I} / \sigma(\mathrm{I})\rangle$ & $23.9(5.5)$ & $24.3(6.9)$ & $12.2(4.0)$ \\
\hline $\mathrm{R}_{\text {merge }}^{3}(\%)$ & $6.6(28.8)$ & $5.4(23.7)$ & $10.8(90.0)$ \\
\hline $\mathrm{R}_{\mathrm{pim}}^{4}(\%)$ & $2.6(12.9)$ & $2.5(10.8)$ & $4.3(0.359)$ \\
\hline $\mathrm{CC}_{1 / 2}{ }^{5}$ & $0.999(0.981)$ & $0.999(0.986)$ & $0.997(0.843)$ \\
\hline Wilson B factor $\left(\AA^{2}\right)$ & 17.1 & & 8.8 \\
\hline
\end{tabular}

\section{Refinement and model statistics}

No. of reflections used

$\mathrm{R}_{\text {work }}{ }^{6}$

0.185

0.172

$\mathrm{R}_{\text {free }}{ }^{7}$

0.255

0.206

RMSD

$$
\begin{aligned}
& \text { Bond lengths }(\AA) \\
& \text { Bond angles }\left({ }^{\circ}\right)
\end{aligned}
$$

0.007

0.009

0.932

1.024

No. of non-H atoms:

Protein

792

835

Ligand

18

14

Water oxygens

73

106

Average B factor $\left(\AA^{2}\right)$ :

Protein atoms

Ligands

46.8

33.5

Water oxygens

35

Ramachandran plot:

Favoured (\%)

100

99

Allowed (\%)

100

0

Outlier (\%)

0

1

PDB code

$5 \mathrm{~V} 23$

$5 \mathrm{~V} 0 \mathrm{M}$

\footnotetext{
${ }^{1}$ Values in parentheses indicate the highest resolution shell
} 
${ }^{2} \mathrm{AU}$, asymmetric unit

${ }^{3} \mathrm{R}_{\text {merge }}=\Sigma_{\mathrm{h}} \Sigma_{\mathrm{i}}\left|\mathrm{I}_{\mathrm{i}}(\mathrm{h})-<\mathrm{I}(\mathrm{h})>\right| / \Sigma_{\mathrm{h}} \Sigma_{\mathrm{i}} \mathrm{I}_{\mathrm{i}}(\mathrm{h})$

${ }^{4} \mathrm{R}_{\mathrm{pim}}=\Sigma_{\mathrm{hkl}}\left\{1 /\left(\mathrm{N}_{\mathrm{hkl}}-1\right)\right\}^{1 / 2} \Sigma_{\mathrm{i}}\left|\mathrm{I}_{\mathrm{i}}(\mathrm{hkl})-<\mathrm{I}(\mathrm{hkl})>\right| / \Sigma_{\mathrm{hkl}} \Sigma_{\mathrm{i}} \mathrm{I}_{\mathrm{i}}(\mathrm{hkl})$

${ }^{5} \mathrm{CC}_{1 / 2}$

${ }^{6} \mathrm{R}_{\text {work }}=\Sigma_{\text {hkl }}|| \mathrm{F}_{\text {obs }}|-| \mathrm{F}_{\text {calc }}|| / \Sigma_{\text {hkl }}\left|\mathrm{F}_{\text {obs }}\right|$

${ }^{7} \mathrm{R}_{\text {free }}$ is the cross-validation $\mathrm{R}$ factor for $5 \%$ of the reflections against which the model was not refined. 
Achtman, M., Neibert, M., Crowe, B.A., Strittmatter, W., Kusecek, B., Weyse, E., Walsh, M.J., Slawig, B., Morelli, G., Moll, A., et al, 1988. Purification and characterization of eight class 5 outer membrane protein variants from a clone of Neisseria meningitidis serogroup A. J Exp Med $168,507-25$.

Aird, W.C., 2012. Endothelial cell heterogeneity. Cold Spring Harb. Perspect. Med. 2, a006429. https://doi.org/10.1101/cshperspect.a006429

Aubey, F., Corre, J.-P., Kong, Y., Xu, X., Obino, D., Goussard, S., Lapeyrere, C., Souphron, J., Couturier, C., Renard, S., Duménil, G., 2019. Inhibitors of the Neisseria meningitidis PilF ATPase provoke type IV pilus disassembly. Proc. Natl. Acad. Sci. U. S. A. 116, 8481-8486. https://doi.org/10.1073/pnas.1817757116

Audry, M., Robbe-Masselot, C., Barnier, J.P., Gachet, B., Saubamea, B., Schmitt, A., Schonherr-Hellec, S., Leonard, R., Nassif, X., Coureuil, M., 2019. Airway Mucus Restricts Neisseria meningitidis Away from Nasopharyngeal Epithelial Cells and Protects the Mucosa from Inflammation. mSphere 4. https://doi.org/10.1128/mSphere.00494-19

Barnier, J.-P., Euphrasie, D., Join-Lambert, O., Audry, M., Schonherr-Hellec, S., Schmitt, T., Bourdoulous, S., Coureuil, M., Nassif, X., El Behi, M., 2021. Type IV pilus retraction enables sustained bacteremia and plays a key role in the outcome of meningococcal sepsis in a humanized mouse model. PLoS Pathog. 17, e1009299. https://doi.org/10.1371/journal.ppat.1009299

Bernard, S.C., Simpson, N., Join-Lambert, O., Federici, C., Laran-Chich, M.-P., Maïssa, N., BouzinbaSégard, H., Morand, P.C., Chretien, F., Taouji, S., Chevet, E., Janel, S., Lafont, F., Coureuil, M., Segura, A., Niedergang, F., Marullo, S., Couraud, P.-O., Nassif, X., Bourdoulous, S., 2014. Pathogenic Neisseria meningitidis utilizes CD147 for vascular colonization. Nat. Med. 20, 725-731. https://doi.org/10.1038/nm.3563

Berry, J.-L., Xu, Y., Ward, P.N., Lea, S.M., Matthews, S.J., Pelicic, V., 2016. A Comparative Structure/Function Analysis of Two Type IV Pilin DNA Receptors Defines a Novel Mode of DNA Binding. Struct. Lond. Engl. 1993 24, 926-934. https://doi.org/10.1016/j.str.2016.04.001

Biais, N., Higashi, D.L., Brujic, J., So, M., Sheetz, M.P., 2010. Force-dependent polymorphism in type IV pili reveals hidden epitopes. Proc Natl Acad Sci U A 107, 11358-63. https://doi.org/10.1073/pnas.0911328107

Brissac, T., Mikaty, G., Dumenil, G., Coureuil, M., Nassif, X., 2012. The meningococcal minor pilin PilX is responsible for type IV pilus conformational changes associated with signaling to endothelial cells. Infect Immun 80, 3297-306. https://doi.org/10.1128/IAI.00369-12

Cahoon, L.A., Seifert, H.S., 2009. An alternative DNA structure is necessary for pilin antigenic variation in Neisseria gonorrhoeae. Science 325, 764-7. https://doi.org/10.1126/science.1175653

Capel, E., Barnier, J.-P., Zomer, A.L., Bole-Feysot, C., Nussbaumer, T., Jamet, A., Lécuyer, H., Euphrasie, D., Virion, Z., Frapy, E., Pélissier, P., Join-Lambert, O., Rattei, T., Bourdoulous, S., Nassif, X., Coureuil, M., 2017. Peripheral blood vessels are a niche for blood-borne meningococci. Virulence 8, 1808-1819. https://doi.org/10.1080/21505594.2017.1391446

Cehovin, A., Kroll, J.S., Pelicic, V., 2011. Testing the vaccine potential of PilV, PilX and ComP, minor subunits of Neisseria meningitidis type IV pili. Vaccine.

Cehovin, A., Simpson, P.J., McDowell, M.A., Brown, D.R., Noschese, R., Pallett, M., Brady, J., Baldwin, G.S., Lea, S.M., Matthews, S.J., Pelicic, V., 2013. Specific DNA recognition mediated by a type IV pilin. Proc Natl Acad Sci U A 110, 3065-70. https://doi.org/10.1073/pnas.1218832110

Cehovin, A., Winterbotham, M., Lucidarme, J., Borrow, R., Tang, C.M., Exley, R.M., Pelicic, V., 2010. Sequence conservation of pilus subunits in Neisseria meningitidis. Vaccine 28, 4817-26. https://doi.org/10.1016/j.vaccine.2010.04.065

Chang, Q., Tzeng, Y.L., Stephens, D.S., 2012. Meningococcal disease: changes in epidemiology and prevention. Clin Epidemiol 4, 237-45. https://doi.org/10.2147/CLEP.S28410 
Christensen, H., May, M., Bowen, L., Hickman, M., Trotter, C.L., 2010. Meningococcal carriage by age: a systematic review and meta-analysis. Lancet Infect. Dis. 10, 853-861. https://doi.org/10.1016/S1473-3099(10)70251-6

Coureuil, M., Jamet, A., Bille, E., Lécuyer, H., Bourdoulous, S., Nassif, X., 2019. Molecular interactions between Neisseria meningitidis and its human host. Cell. Microbiol. 21, e13063. https://doi.org/10.1111/cmi.13063

Craig, L., Forest, K.T., Maier, B., 2019. Type IV pili: dynamics, biophysics and functional consequences. Nat. Rev. Microbiol. 17, 429-440. https://doi.org/10.1038/s41579-019-0195-4

Craig, L., Volkmann, N., Arvai, A.S., Pique, M.E., Yeager, M., Egelman, E.H., Tainer, J.A., 2006. Type IV pilus structure by cryo-electron microscopy and crystallography: implications for pilus assembly and functions. Mol Cell 23, 651-62. https://doi.org/10.1016/j.molcel.2006.07.004

Crooks, G.E., Hon, G., Chandonia, J.-M., Brenner, S.E., 2004. WebLogo: a sequence logo generator. Genome Res. 14, 1188-1190. https://doi.org/10.1101/gr.849004

Denis, K., Le Bris, M., Le Guennec, L., Barnier, J.-P., Faure, C., Gouge, A., Bouzinba-Ségard, H., Jamet, A., Euphrasie, D., Durel, B., Barois, N., Pelissier, P., Morand, P.C., Coureuil, M., Lafont, F., JoinLambert, O., Nassif, X., Bourdoulous, S., 2019. Targeting Type IV pili as an antivirulence strategy against invasive meningococcal disease. Nat. Microbiol. 4, 972-984. https://doi.org/10.1038/s41564-019-0395-8

Dyer, D.W., McKenna, W., Woods, J.P., Sparling, P.F., 1987. Isolation by streptonigrin enrichment and characterization of a transferrin-specific iron uptake mutant of Neisseria meningitidis. Microb. Pathog. 3, 351-363. https://doi.org/10.1016/0882-4010(87)90005-2

Fu, L., Niu, B., Zhu, Z., Wu, S., Li, W., 2012. CD-HIT: accelerated for clustering the next-generation sequencing data. Bioinforma. Oxf. Engl. 28, 3150-3152. https://doi.org/10.1093/bioinformatics/bts565

Helaine, S., Carbonnelle, E., Prouvensier, L., Beretti, J.L., Nassif, X., Pelicic, V., 2005. PilX, a pilusassociated protein essential for bacterial aggregation, is a key to pilus-facilitated attachment of Neisseria meningitidis to human cells. Mol Microbiol 55, 65-77.

Helaine, S., Dyer, D.H., Nassif, X., Pelicic, V., Forest, K.T., 2007. 3D structure/function analysis of PilX reveals how minor pilins can modulate the virulence properties of type IV pili. Proc. Natl. Acad. Sci. U. S. A. 104, 15888-15893. https://doi.org/10.1073/pnas.0707581104

Imhaus, A.F., Dumenil, G., 2014. The number of Neisseria meningitidis type IV pili determines host cell interaction. Embo J. https://doi.org/10.15252/embj.201488031

Join-Lambert, O., Lecuyer, H., Miller, F., Lelievre, L., Jamet, A., Furio, L., Schmitt, A., Pelissier, P., Fraitag, S., Coureuil, M., Nassif, X., 2013. Meningococcal Interaction to Microvasculature Triggers the Tissular Lesions of Purpura Fulminans. J. Infect. Dis. 208, 1590-1597. https://doi.org/10.1093/infdis/jit301

Kolappan, S., Coureuil, M., Yu, X., Nassif, X., Egelman, E.H., Craig, L., 2016. Structure of the Neisseria meningitidis Type IV pilus. Nat. Commun. 7, 13015. https://doi.org/10.1038/ncomms13015

Kolappan, S., Ng, D., Yang, G., Harn, T., Craig, L., 2015. Crystal Structure of the Minor Pilin CofB, the Initiator of CFA/III Pilus Assembly in Enterotoxigenic Escherichia coli. J. Biol. Chem. 290, 25805-25818. https://doi.org/10.1074/jbc.M115.676106

Le Guennec, L., Virion, Z., Bouzinba-Ségard, H., Robbe-Masselot, C., Léonard, R., Nassif, X., Bourdoulous, S., Coureuil, M., 2020. Receptor recognition by meningococcal type IV pili relies on a specific complex N-glycan. Proc. Natl. Acad. Sci. U. S. A. 117, 2606-2612. https://doi.org/10.1073/pnas.1919567117

Li, J., Egelman, E., Craig, L., 2012. Electron microscopy reconstruction of the Vibrio cholerae toxin coregulated pilus and comparative analysis with the Neisseria gonorrhoeae GC pilus. J Mol Biol 418, 47-64.

Maïssa, N., Covarelli, V., Janel, S., Durel, B., Simpson, N., Bernard, S.C., Pardo-Lopez, L., BouzinbaSégard, H., Faure, C., Scott, M.G.H., Coureuil, M., Morand, P.C., Lafont, F., Nassif, X., Marullo, S., Bourdoulous, S., 2017. Strength of Neisseria meningitidis binding to endothelial cells 
requires highly-ordered CD147/ $\beta 2$-adrenoceptor clusters assembled by alpha-actinin-4. Nat. Commun. 8, 15764. https://doi.org/10.1038/ncomms15764

McCallum, M., Burrows, L.L., Howell, P.L., 2019. The Dynamic Structures of the Type IV Pilus. Microbiol. Spectr. 7. https://doi.org/10.1128/microbiolspec.PSIB-0006-2018

Mikaty, G., Soyer, M., Mairey, E., Henry, N., Dyer, D., Forest, K.T., Morand, P., Guadagnini, S., Prévost, M.C., Nassif, X., Duménil, G., 2009. Extracellular bacterial pathogen induces host cell surface reorganization to resist shear stress. PLoS Pathog. 5, e1000314. https://doi.org/10.1371/journal.ppat.1000314

Muttalif, A.R., Presa, J.V., Haridy, H., Gamil, A., Serra, L.C., Cané, A., 2019. Incidence and Prevention of Invasive Meningococcal Disease in Global Mass Gathering Events. Infect. Dis. Ther. 8, 569579. https://doi.org/10.1007/s40121-019-00262-9

Ng, D., Harn, T., Altindal, T., Kolappan, S., Marles, J.M., Lala, R., Spielman, I., Gao, Y., Hauke, C.A., Kovacikova, G., Verjee, Z., Taylor, R.K., Biais, N., Craig, L., 2016. The Vibrio cholerae Minor Pilin TcpB Initiates Assembly and Retraction of the Toxin-Coregulated Pilus. PLoS Pathog. 12, e1006109. https://doi.org/10.1371/journal.ppat.1006109

Nguyen, Y., Sugiman-Marangos, S., Harvey, H., Bell, S.D., Charlton, C.L., Junop, M.S., Burrows, L.L., 2015. Pseudomonas aeruginosa minor pilins prime type IVa pilus assembly and promote surface display of the PilY1 adhesin. J. Biol. Chem. 290, 601-611. https://doi.org/10.1074/jbc.M114.616904

Parge, H.E., Forest, K.T., Hickey, M.J., Christensen, D.A., Getzoff, E.D., Tainer, J.A., 1995. Structure of the fibre-forming protein pilin at 2.6 A resolution. Nature 378, 32-8.

Ramsey, M.E., Hackett, K.T., Kotha, C., Dillard, J.P., 2012. New Complementation Constructs for Inducible and Constitutive Gene Expression in Neisseria gonorrhoeae and Neisseria meningitidis. Appl. Environ. Microbiol. 78, 3068-3078. https://doi.org/10.1128/AEM.0787111

Siena, E., Bodini, M., Medini, D., 2018. Interplay Between Virulence and Variability Factors as a Potential Driver of Invasive Meningococcal Disease. Comput. Struct. Biotechnol. J. 16, 61-69. https://doi.org/10.1016/j.csbj.2018.02.002

Sievers, F., Higgins, D.G., 2014. Clustal omega. Curr. Protoc. Bioinforma. 48, 3.13.1-16. https://doi.org/10.1002/0471250953.bi0313s48

Takahashi, H., Yanagisawa, T., Kim, K.S., Yokoyama, S., Ohnishi, M., 2012. Meningococcal PilV potentiates Neisseria meningitidis type IV pilus-mediated internalization into human endothelial and epithelial cells. Infect Immun 80, 4154-66. https://doi.org/10.1128/IAI.00423-12

Treuner-Lange, A., Chang, Y.-W., Glatter, T., Herfurth, M., Lindow, S., Chreifi, G., Jensen, G.J., Søgaard-Andersen, L., 2020. PilY1 and minor pilins form a complex priming the type IVa pilus in Myxococcus xanthus. Nat. Commun. 11, 5054. https://doi.org/10.1038/s41467-02018803-z

Van Duyne, G.D., Standaert, R.F., Karplus, P.A., Schreiber, S.L., Clardy, J., 1993. Atomic structures of the human immunophilin FKBP-12 complexes with FK506 and rapamycin. J. Mol. Biol. 229, 105-124. https://doi.org/10.1006/jmbi.1993.1012

Vink, C., Rudenko, G., Seifert, H.S., 2012. Microbial antigenic variation mediated by homologous DNA recombination. FEMS Microbiol. Rev. 36, 917-948. https://doi.org/10.1111/j.15746976.2011.00321.x

Virion, Z., Doly, S., Saha, K., Lambert, M., Guillonneau, F., Bied, C., Duke, R.M., Rudd, P.M., RobbeMasselot, C., Nassif, X., Coureuil, M., Marullo, S., 2019. Sialic acid mediated mechanical activation of $\beta 2$ adrenergic receptors by bacterial pili. Nat. Commun. 10, 4752. https://doi.org/10.1038/s41467-019-12685-6

Wang, F., Coureuil, M., Osinski, T., Orlova, A., Altindal, T., Gesbert, G., Nassif, X., Egelman, E.H., Craig, L., 2017. Cryoelectron Microscopy Reconstructions of the Pseudomonas aeruginosa and Neisseria gonorrhoeae Type IV Pili at Sub-nanometer Resolution. Struct. Lond. Engl. 1993 25, 1423-1435.e4. https://doi.org/10.1016/j.str.2017.07.016 
Weksler, B.B., Subileau, E.A., Perriere, N., Charneau, P., Holloway, K., Leveque, M., Tricoire-Leignel, H., Nicotra, A., Bourdoulous, S., Turowski, P., Male, D.K., Roux, F., Greenwood, J., Romero, I.A., Couraud, P.O., 2005. Blood-brain barrier-specific properties of a human adult brain endothelial cell line. Faseb J 19, 1872-4.

Winther-Larsen, H.C., Hegge, F.T., Wolfgang, M., Hayes, S.F., van Putten, J.P., Koomey, M., 2001. Neisseria gonorrhoeae PilV, a type IV pilus-associated protein essential to human epithelial cell adherence. Proc Natl Acad Sci U A 98, 15276-81.

Winther-Larsen, H.C., Wolfgang, M., Dunham, S., van Putten, J.P.M., Dorward, D., Løvold, C., Aas, F.E., Koomey, M., 2005. A conserved set of pilin-like molecules controls type IV pilus dynamics and organelle-associated functions in Neisseria gonorrhoeae. Mol. Microbiol. 56, 903-917. https://doi.org/10.1111/j.1365-2958.2005.04591.x

Wolfgang, M., Lauer, P., Park, H.S., Brossay, L., Hebert, J., Koomey, M., 1998. PilT mutations lead to simultaneous defects in competence for natural transformation and twitching motility in piliated Neisseria gonorrhoeae. Mol Microbiol 29, 321-30.

Wörmann, M.E., Horien, C.L., Bennett, J.S., Jolley, K.A., Maiden, M.C.J., Tang, C.M., Aho, E.L., Exley, R.M., 2014. Sequence, distribution and chromosomal context of class I and class II pilin genes of Neisseria meningitidis identified in whole genome sequences. BMC Genomics 15, 253. https://doi.org/10.1186/1471-2164-15-253 
FIGURE 1
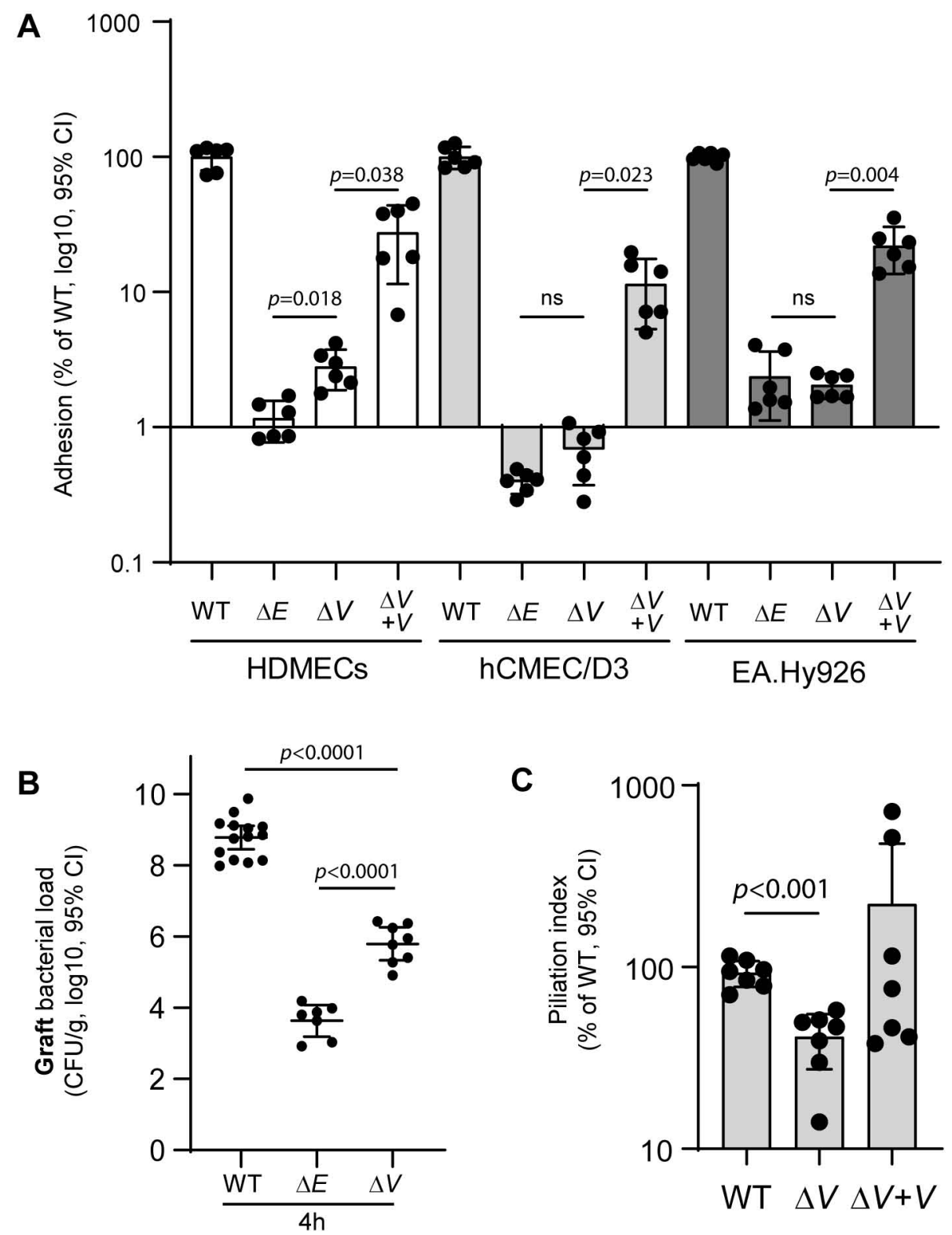
FIGURE 2
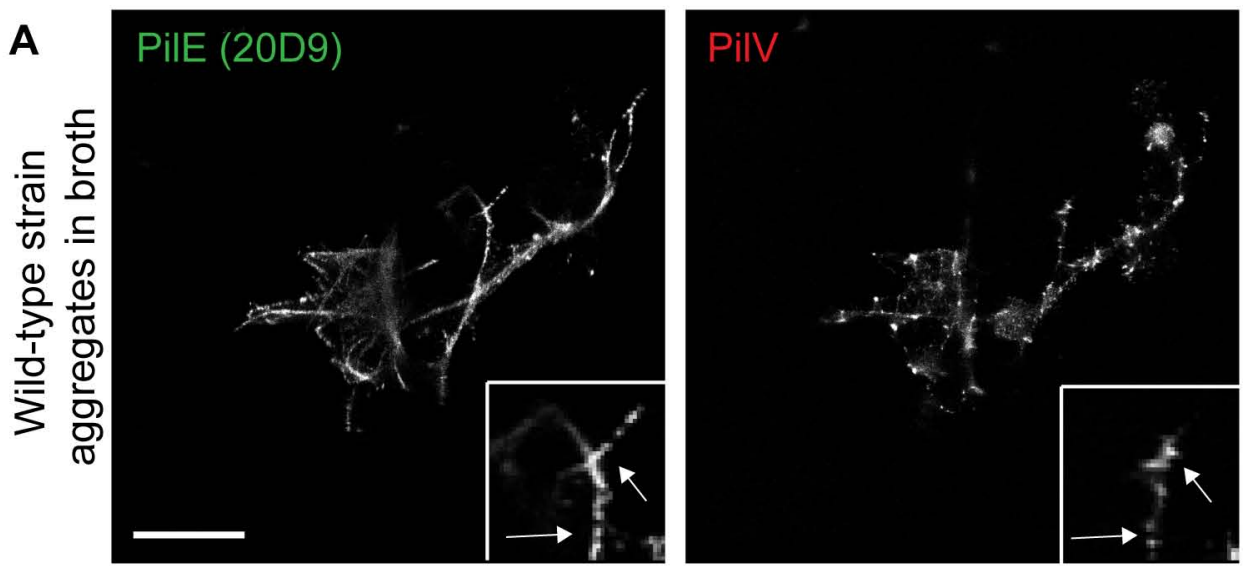

merge
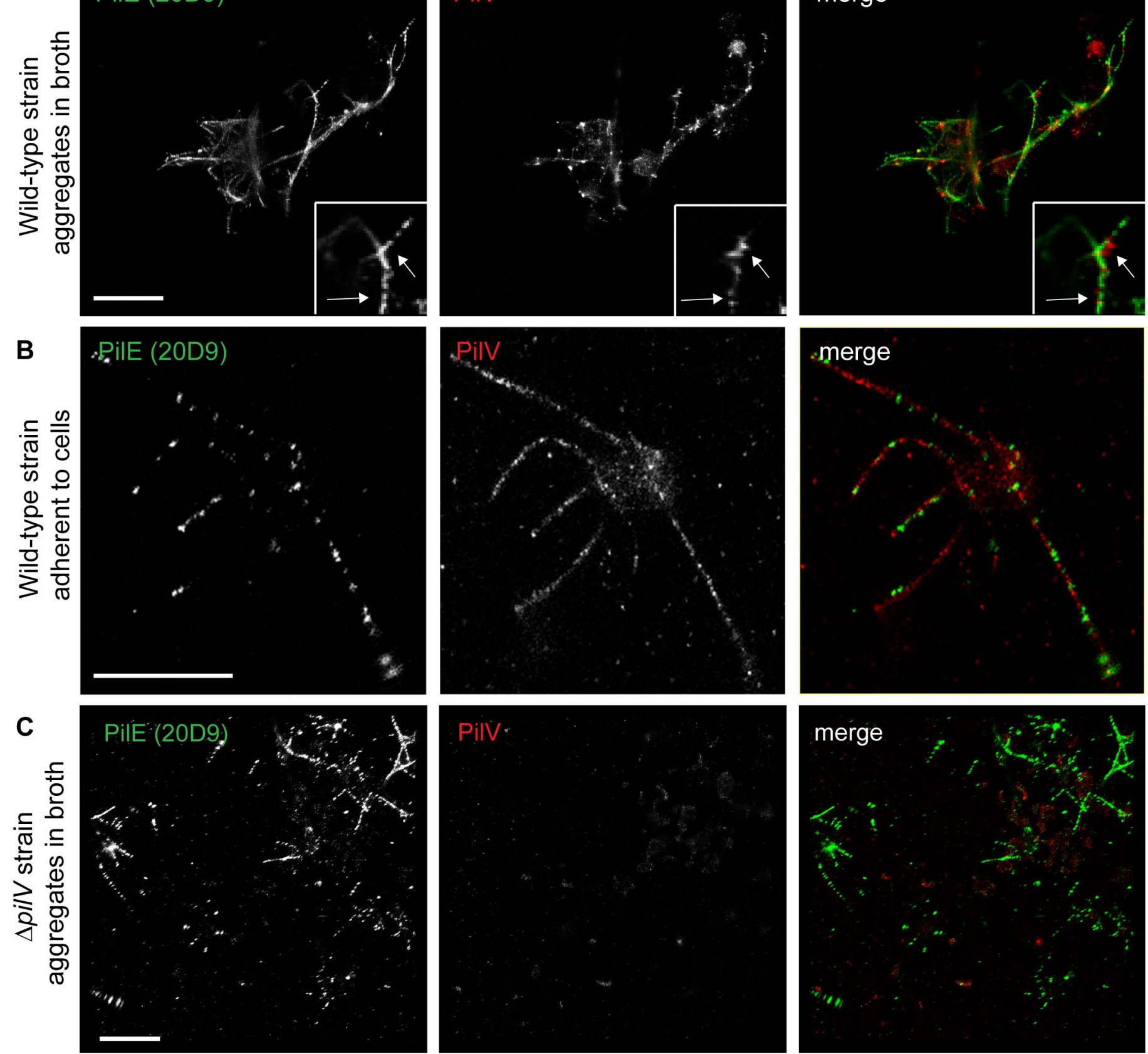

PilV.

merge

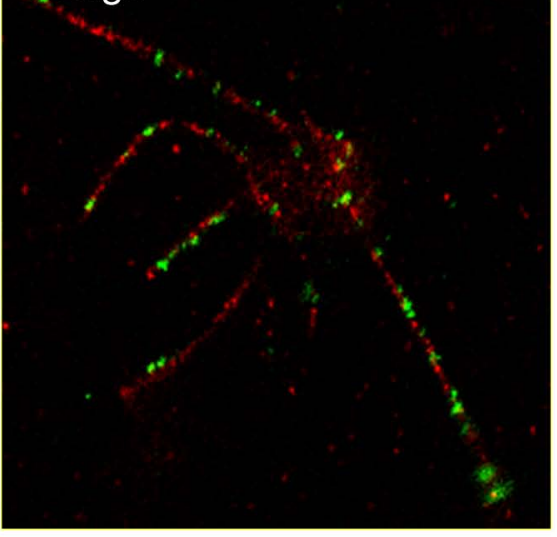

merge

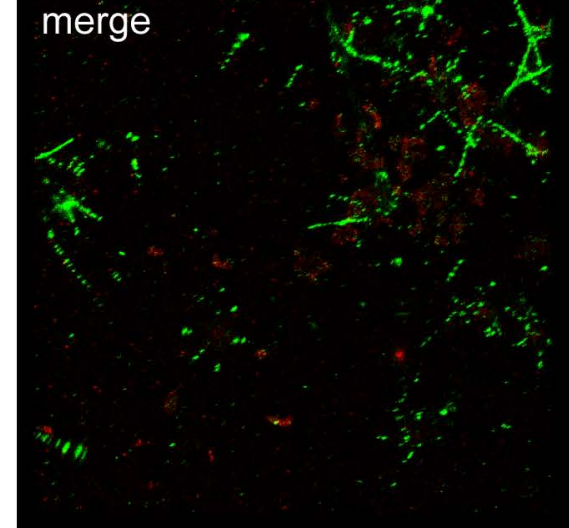



A Nm PIIE FTLIELMIVIAIV ILAAVAL AYODYTARAOVSEAILLAEFOKSAVTEYYLNHG 55

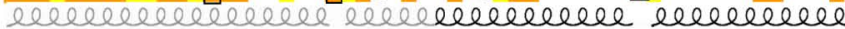

$$
\longleftrightarrow \alpha \beta-100 p \longrightarrow
$$

$$
\text { B2 }
$$

Nm PilV TFKT----- YDKNKLKQNKYFNVTLSKVSPDHFTLQADPNPTTNDGETC-VVTL 103 Nm PilE EWPGDNSSAGVATSADIKGKYVQSVTVA-NG--VITAQMASSNVNNEIKSKKLSL 107 $\beta 4$

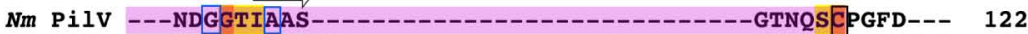
Nm PIIE WAKRQNGSVKWFCGQPVTRTTATATDVAAANGKTDDKINTKHLPSTCRDDSSAS 161

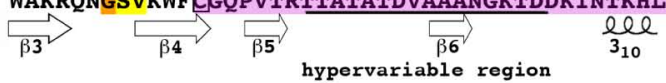

\section{$\mathbf{E}$}

$14 \quad 22 \quad 42$

Nm PIIV FTLLELMIAVAIL ILTLITY SYKTYIRRVRLSEVRTTLLHNAQTMERYYRQKG 55 Nm PilE FTLIELMIVIAIV ILAAVAL AYQDYTARAQVSEAILLAEGQKSAVTEYYLNHG 55 Nm PilX FTLIEMMIVVAIL IISVIAI SYOSYIEKGYOSOLYTEMVGINNISKOFILKNP 55 $N m$ COMP FTLVELISVVLILSVLALIVY SYRNYVEKAKINAVRAALLENAHFMEKFYLQNG 55 Ng PilE FTLIELMIVIAIV ILAAVAL AYQDYTARAOVSEAILLAEGOKSAVTEYYLNHG 55

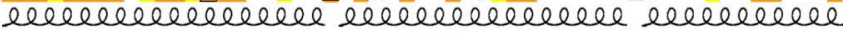
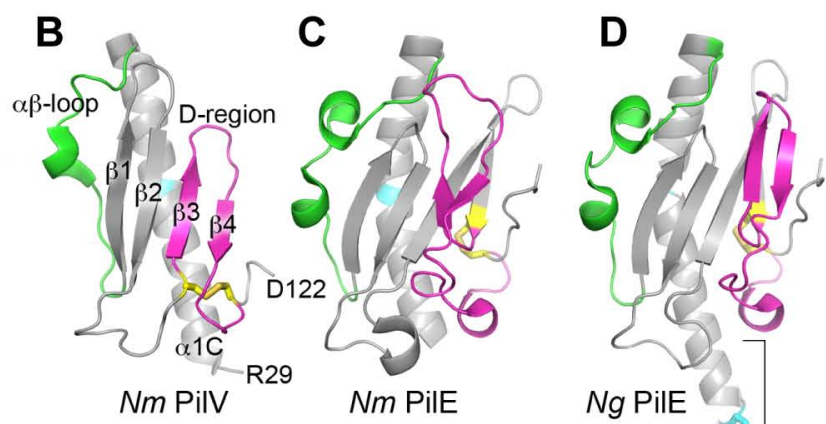

Ng PilE

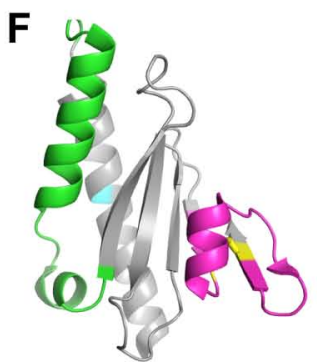

Nm PilX

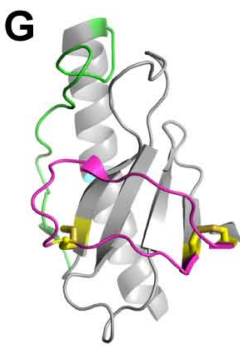

Nm ComP
H

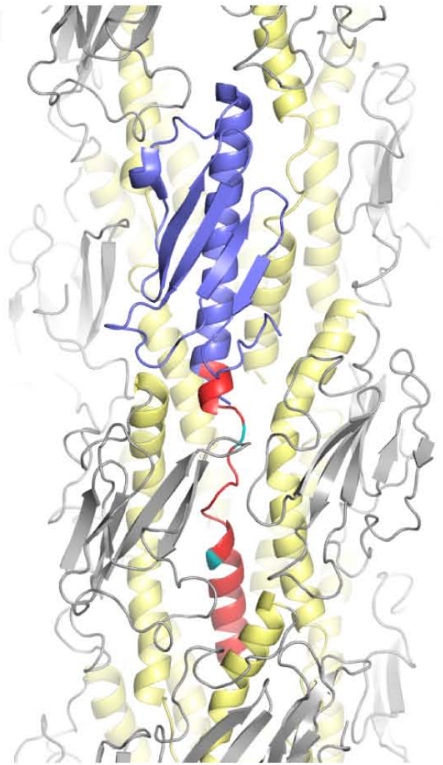

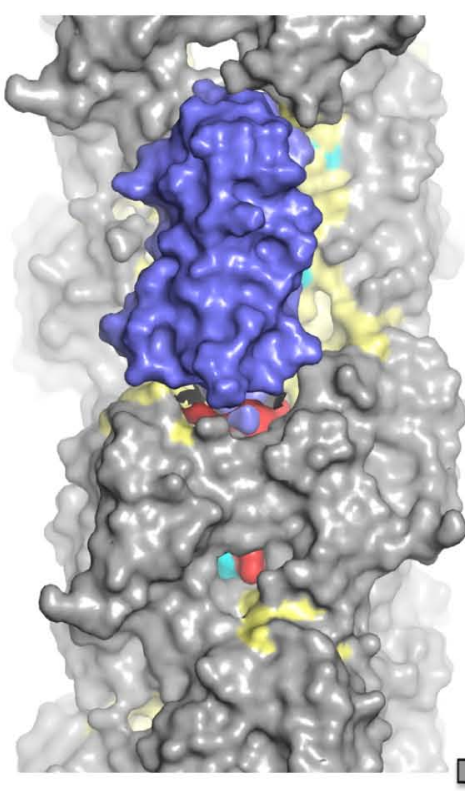

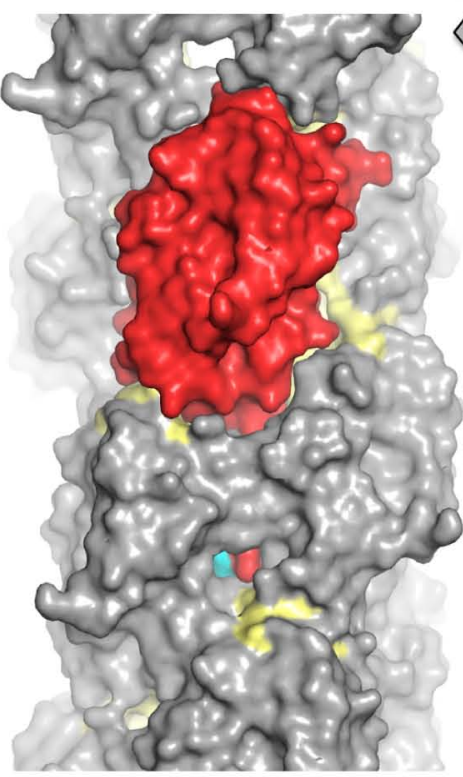

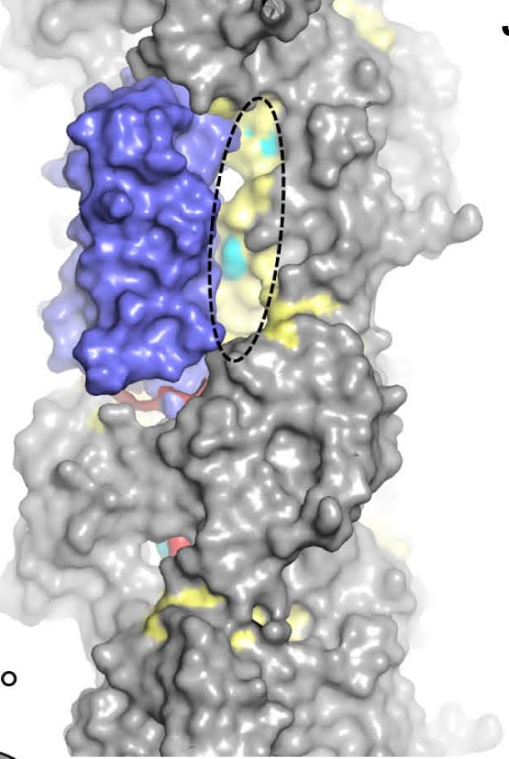

$\mathrm{J}$
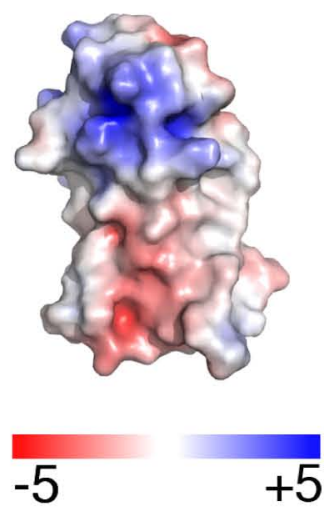

K

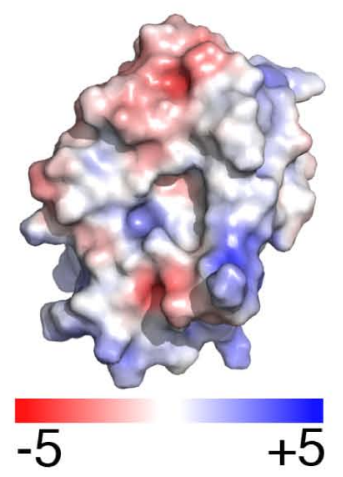


A scanning mutagenesis step one

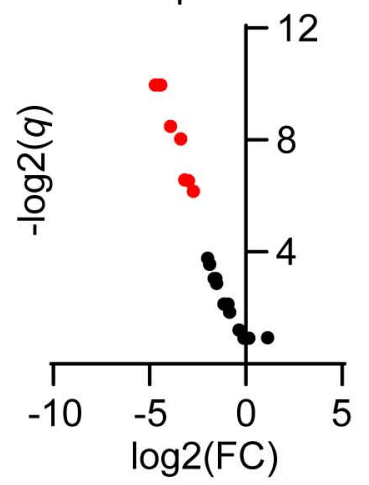

D

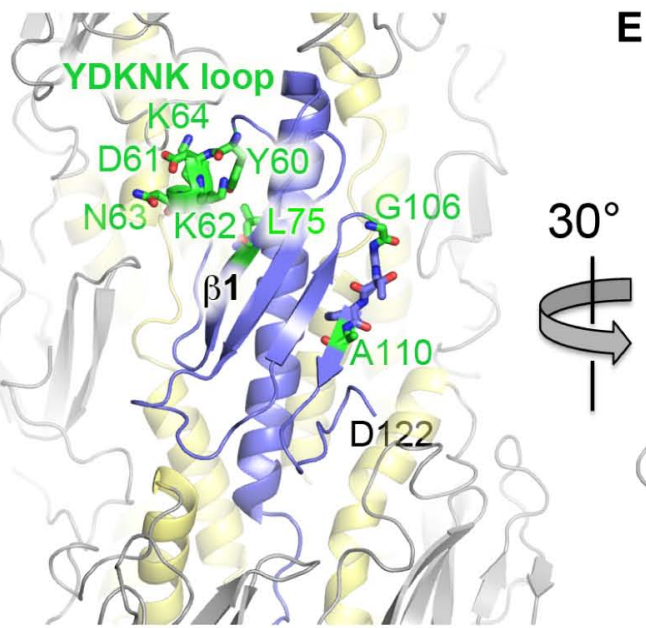

G

PilV2C4.3 1 FT LLELM I AVA I LG I LTL I TYPSYKTY I RRVR 32

PilVFam18

PilVz2491

consensus

(PubMLST)

PilV2C4.3

PilVfam18

PilVz2491

consensus

(PubMLST)

PilV2C4.3

PilVFAm18

PilVz2491

consensus

PilV2C4.3

PilVFam18

PilVz2491
B step two

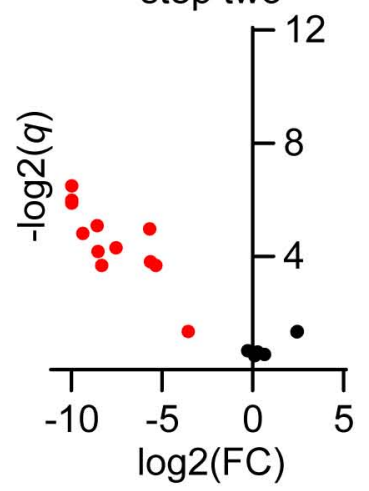
scanning mutagenesis
E

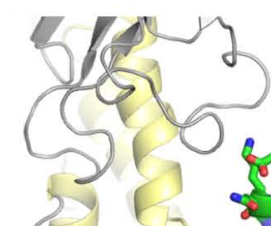
FTLLELM I AVA I LG I LTL I TYPSYKTY IRR IR FTLLELM I AVA I LGILTL I TYPSYKTY IRRVR FTLLELM I AVA I LG I LTLI TYPSYKTY IRRVR LSEVRTTLLHNAQTMERYYRQKGTFKT YDKNK 64 LSEVKS TLLMNAQTMERYYRQKGT FEN YDKNK LSEVKS TLLMNAQTMERYYRQKGTFATYDKNK LSEVKS TLLMNAQTMERYYRQKGTFENYDKNK LKQNKYFNVTLSKVSPDHFTLQADPNPTTNDG 96 LKQNKYFNVTLSKVSPDHFTLQADPNPT TNDG LKQNEYFK I TLSEVSPDHFTLQAVPDPATNEG LKQNKYFN I TLSKVSPDHFTLQAAPNPTTNDG ETCVVTLNDGGT I AASGTN - QSCPGFD 122 ETCVVTLNDGGT IAASGTN - QSCPGFD ETC IVTLNDGGTLSAAG - NGQSCPDFD ETCVVTLNDGGT I AASGTN-QSCPGFD $0 \%$ 100\%
C

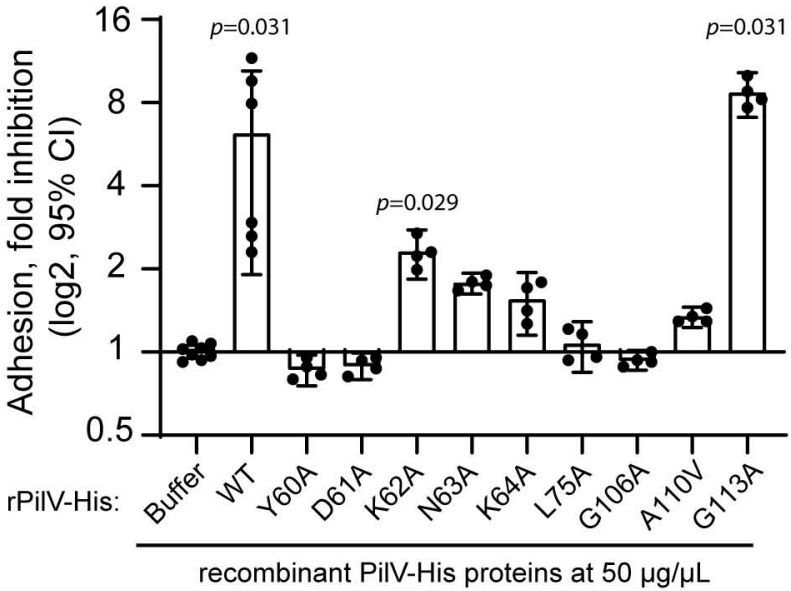

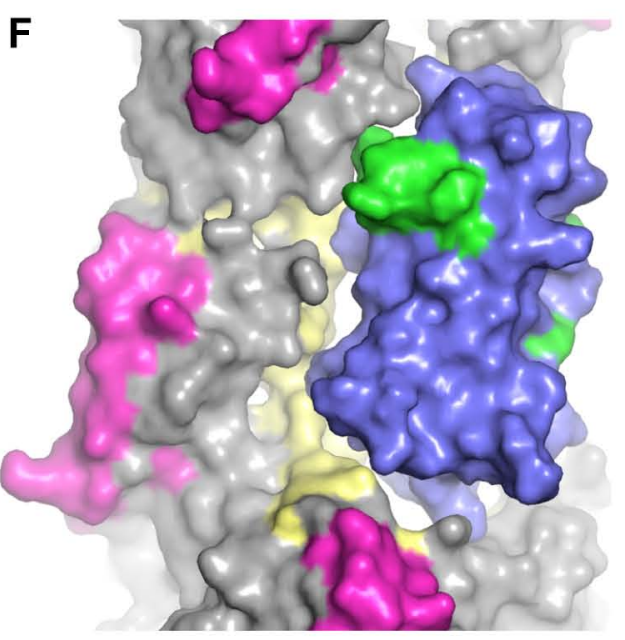

$\mathbf{H}$

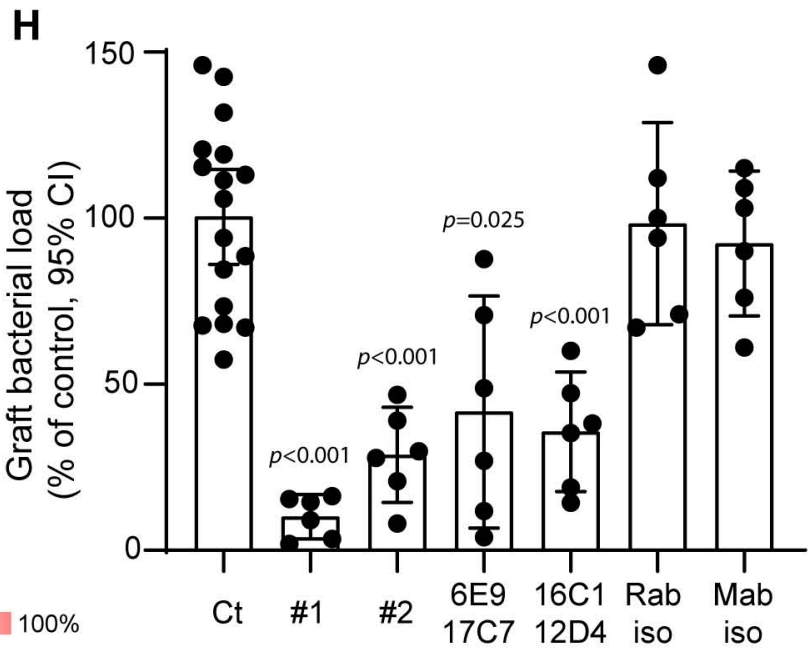




\section{SI APPENDIX}

The minor pilin PilV provides a conserved adhesion site throughout the antigenically variable meningococcal Type IV pilus.

Correspondence to lisa_craig@sfu.ca or mathieu.coureuil@inserm.fr

Page 2: Supplemental figure Legends

Page 6 : Supplemental figure 1

Page 7 : Supplemental figure 2

Page 8 : Supplemental figure 3

Page 9 : Supplemental figure 4

Page 10 : Supplemental figure 5

Page 11 : Supplemental figure 6

Page 12 : Table S2

Page 13 : Table S3

Page 17 : Supplemental Methods 


\section{SUPPLEMENTAL FIGURES LEGENDS}

\section{Supplemental Figure 1. Effect of pilV and pilE mutations on bacteremia, PilV expression}

and piliation. (A) Bacteremia in bloodstream of human skin grafted SCID mice infected intravenously with $5 \times 10^{6} \mathrm{~N}$. meningitidis (WT; $\Delta p i l E, \Delta E ; \Delta p i l V, \Delta V$ ). Bacteremia, quantified by serial dilution on GCB agar plates, was determined at 1 hour and 4 hours after infection. Two independent experiments were performed using a skin batch from a different donor. Each dot represents a single mouse; data are expressed as $\log 10$ of the mean $\pm 95 \% \mathrm{CI}$ of CFU $/ \mathrm{mL}$. Data were analyzed using Bonferroni's multiple comparisons. ns: no statistical difference. (B) Pili of WT, $\Delta V$ and $\Delta$ pilV complemented strain $(\Delta V+V)$ were sheared from the bacterial cells by vortexing and then precipitated with ammonium sulfate (AS). The sheared pilus fraction, the bacteria fraction and whole bacterial lysate were analyzed by SDS-PAGE and immunoblotting with anti-PilE or anti-PilV antibodies. Total proteins were detected in the gel by Stain-Free technology. Images are representative of three experiments made in duplicates or triplicates.

Supplemental Figure 2. PilV and PilE expression and co-locallization. (A) Anti-PilE, antiPilV, anti-NADP-glutamate-deshydrogenase (NADPGH, cytosolic marker) and anti-Rmp4 (membrane marker) immunoblots of sheared ammonium sulfate-precipitated (AS) pili from WT and $\Delta$ pilV strains and immunoblots of whole bacterial lysate from WT. Representative results are shown. $(\mathbf{B}, \mathbf{C})$ Co-immunoprecipitation of PilV and PilE in the sheared pili fraction. Sheared pili from $N$. meningitidis wild-type (WT) and $\Delta p i l V$ were immunoprecipitated with anti-PilE or anti-PilV antibodies and analyzed by SDS-PAGE and immunoblotting with antiPilE or anti-PilV antibodies.

Supplemental Figure 3. Superposition of rPilV on a PilE subunit in the N. meningitidis 
T4P reconstruction. $\mathrm{rPilV}$ (blue) was superimposed onto PilE (red) by aligning residues at the $\mathrm{N}$ - and C-terminal ends of $\alpha \mathrm{C} 1$. Residues at positions 14, 22 and 42 are colored cyan. With the exception of PilV His42, these residues are Gly/Pro.

\section{Supplemental Figure 4. Adhesion, sequence conservation and expression of recombinant}

PilV. (A) Human endothelial cells (hCMEC/D3) were incubated with varying amounts of Histagged recombinant PilV (rPilV-His) prior to infection with WT N. meningitidis. Following infection, unbound bacteria were removed by washes and adherent bacteria were quantified by plating on GCB agar plates and counting CFUs after overnight growth. Adhesion is expressed as the mean $\pm 95 \% \mathrm{CI}$ of CFUs normalized to the control infection in which no rPilV-His was added (0). Data are shown from two independent experiments. Statistical analysis was performed comparing the various amounts of rPilV with the buffer-only controls (rPilV-His, 0). Data were analysed using one-way ANOVA. (B) The ability of rPilV-His to inhibit bacterial adhesion was examined in comparison with recombinant His-tagged ComP (rComP-His). Gray dots and triangles represent the data obtained using two different batches of rPilV-His. WT and rPilV-His, five independent experiments; rComP-His, two independent experiments. Adhesion is expressed as the mean $\pm 95 \%$ CI of CFUs normalized to the control infection in which no recombinant protein was added. Data were analyzed using Brown-Forsythe and Welch ANOVA. (C) Adhesion of $N$. meningitidis $\Delta V$ and $\Delta V$ complemented with pilV alleles from strain 2C4.3, FAM18 or Z2491. Human endothelial cells (hCMEC/D3) were infected for 30 min and the number of cell-associated bacteria was determined by counting CFUs. Experiments were performed three times in duplicate. Adhesion is expressed as mean $\pm 95 \%$ CI of CFUs normalized to $\mathrm{Pil} \mathrm{V}_{2 \mathrm{C} 4.3}$ adhesion. Statistical analyses were performed against $\mathrm{PilV}_{2 \mathrm{C} 4.3}$ with Brown-Forsythe and Welch ANOVA. (D) The sequence of N. meningitidis PilV is conserved. Gene sequences corresponding to the query N. meningitidis pilV (accession number NEIS0487; 
query was made in March 2020) in the MRF Meningococcus Genome Library (PubMLST) database were retrieved and translated. Redundancy of the corresponding protein sequence dataset was reduced with a 100\% identity threshold yielding 699 clusters. Sequences representative of each cluster were aligned with Clustal Omega v1.2.4 (Sievers and Higgins, 2014) with default parameters (Percent Identity Matrix created by Clustal 2.1 is available in Supplemental Table S2). A graphical representation of the amino acid multiple sequence alignment is shown as a sequence logo generated using Weblogo 3.7.4. Blue: hydrophilic residues; green: neutral residues; black: hydrophobic residues. Residues implicated in host cell adhesion are boxed in green. (E) Recombinant His-tagged rPilV (residues 29-122) and alanine/valine-substituted mutants were produced in E. coli. Proteins were analyzed by SDSPAGE and Coomassie Blue staining.

Supplemental Figure 5. Expression and incorporation of PilV F1A and E5A mutants. (A) Anti-PilE, anti-PilV, anti-NADP-glutamate-deshydrogenase (NADPGH, cytosolic marker) immunoblots of whole cell lysates and sheared ammonium sulfate-precipitated (AS) pili from WT and $\Delta$ pilV strains expressing PilV wild type (WT) or PilV mutants.

Supplemental Figure 6. Bacterial loads in bloodstream of human-skin grafted SCID mice injected intravenously with buffer or anti-PilV antibodies. Mice were injected with buffer (control), $100 \mu \mathrm{g}$ of anti-PilV rabbit polyclonal IgG antibodies (\#1, \#2), $100 \mu \mathrm{g}$ of anti-PilV peptide mouse monoclonal IgG antibodies (raised against the $\mathrm{P}_{80}$ DHFTLQADPNPTTNDGE $97_{7}$ peptide: $6 \mathrm{E} 9+17 \mathrm{C} 7,16 \mathrm{C} 1+12 \mathrm{D} 4)$ or their respective isotype control antibodies, followed by infection with $5 \times 10^{5} \mathrm{CFU}$ of WT meningococci. Blood bacterial loads were determined 4 hours after infection and expressed as CFU per ml. Each dot represents a single mouse; data are expressed as the mean $\pm 95 \% \mathrm{CI}$ of $\mathrm{CFU} / \mathrm{ml}$. Statistical analysis were performed against the 
control with ANOVA. No statistical significance.

Supplemental Table S1: PilV identity matrix (separate file)

Supplemental Table S2: Effect of PilV single amino acid substitutions on bacterial adhesion, recombinant pilin expression and inhibition of adhesion.

Supplemental Table S3: Bacterial strains, plasmids and primers.

Supplemental statistics: Statistical analysis and descriptive statistics (separate file) Supplemental methods 
Supplemental figure 1

A

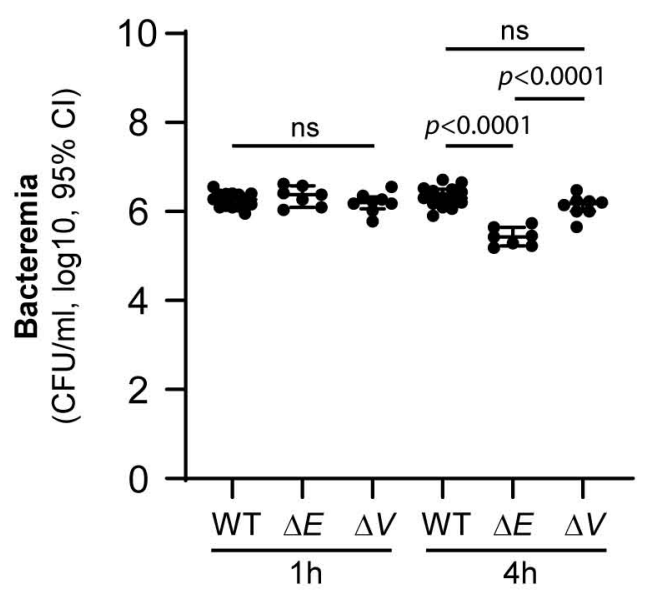

B

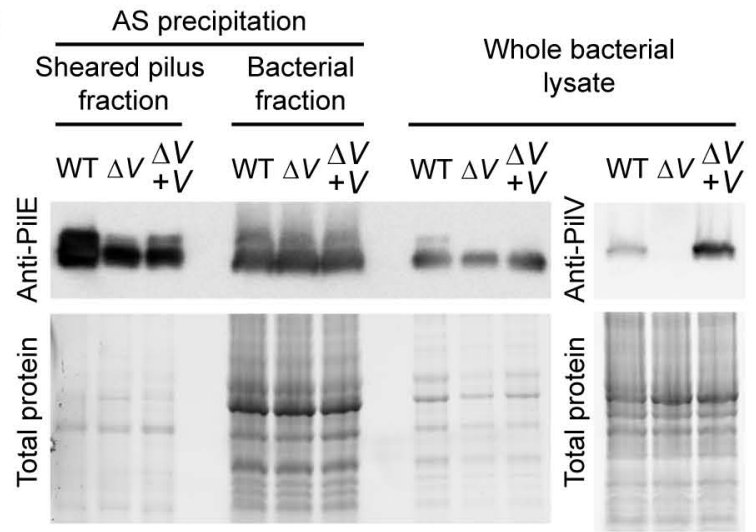


Supplemental figure 2

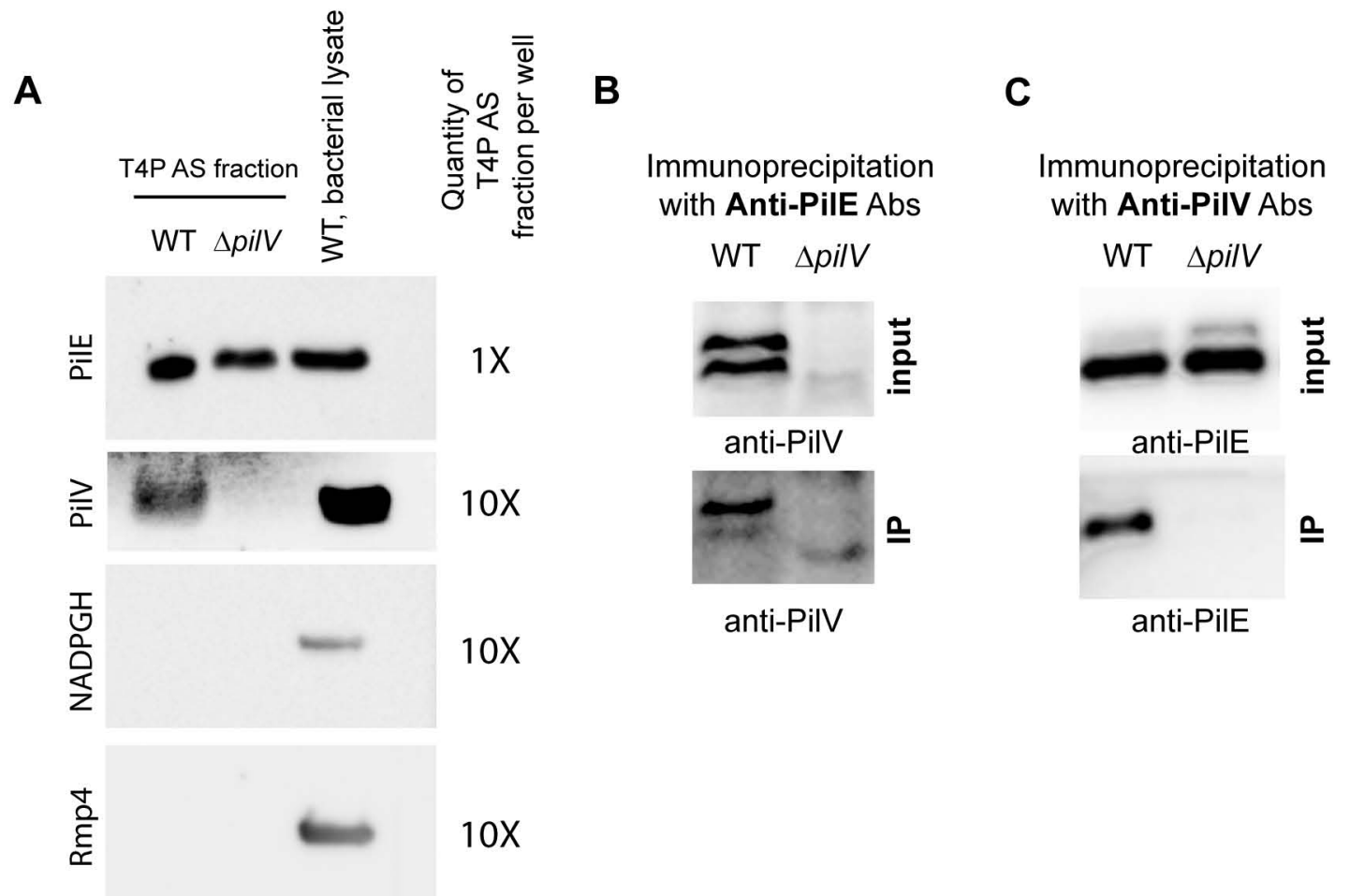


Supplemental figure 3

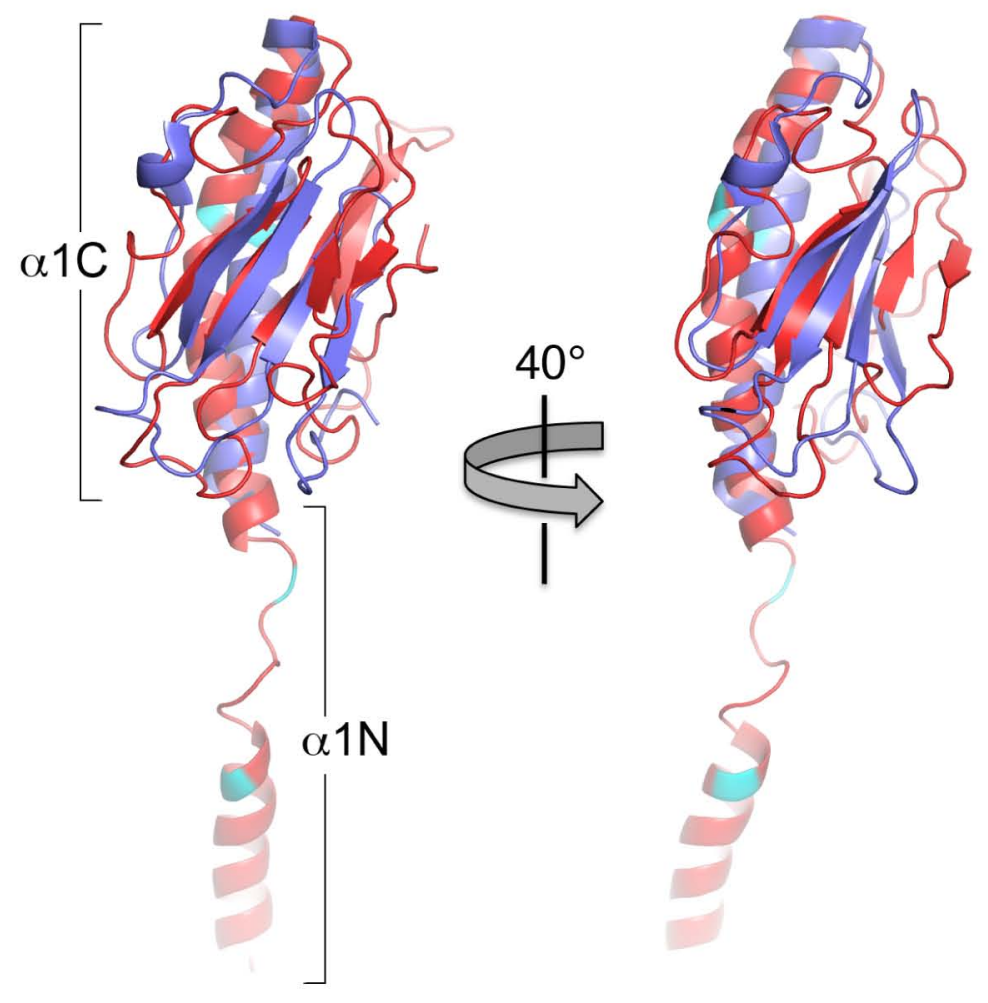


Supplemental figure 4

A

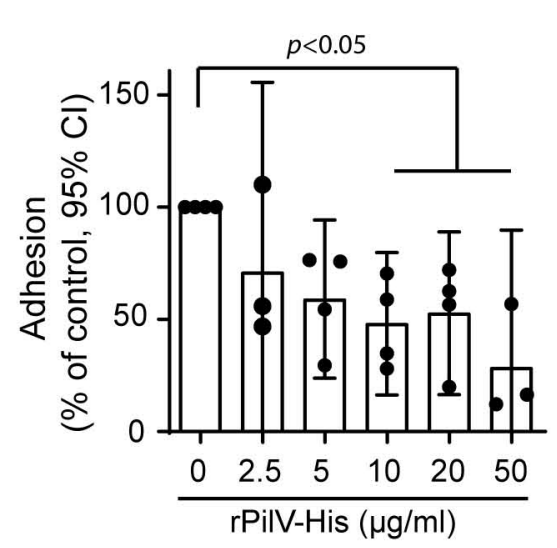

B

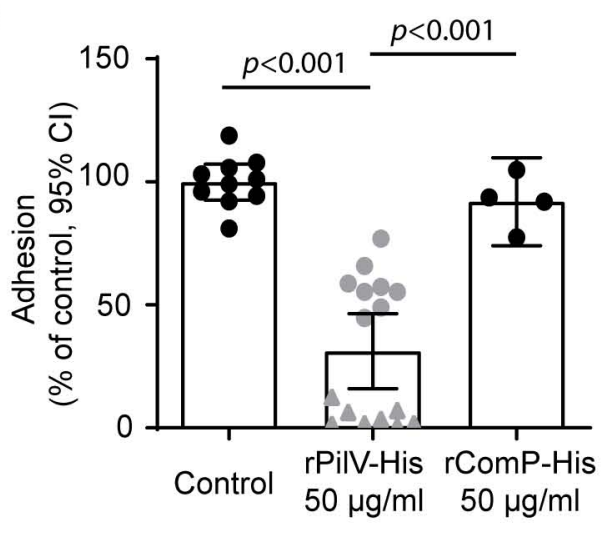

C

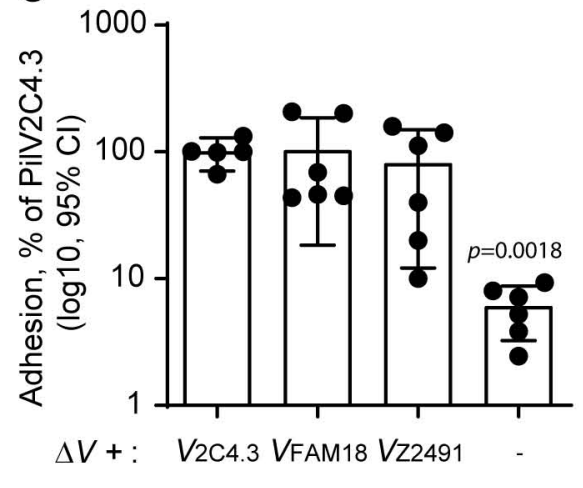

D
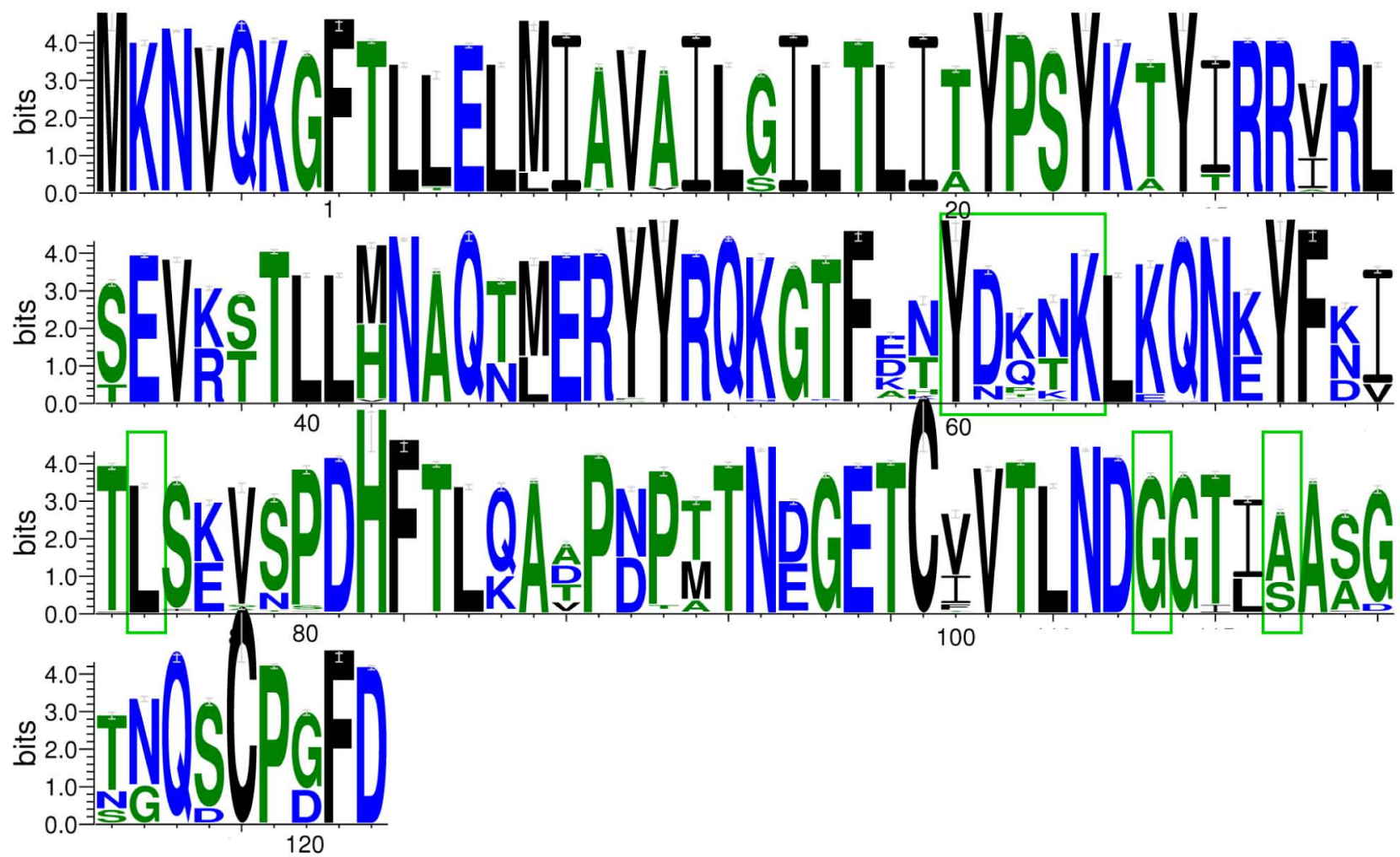

E

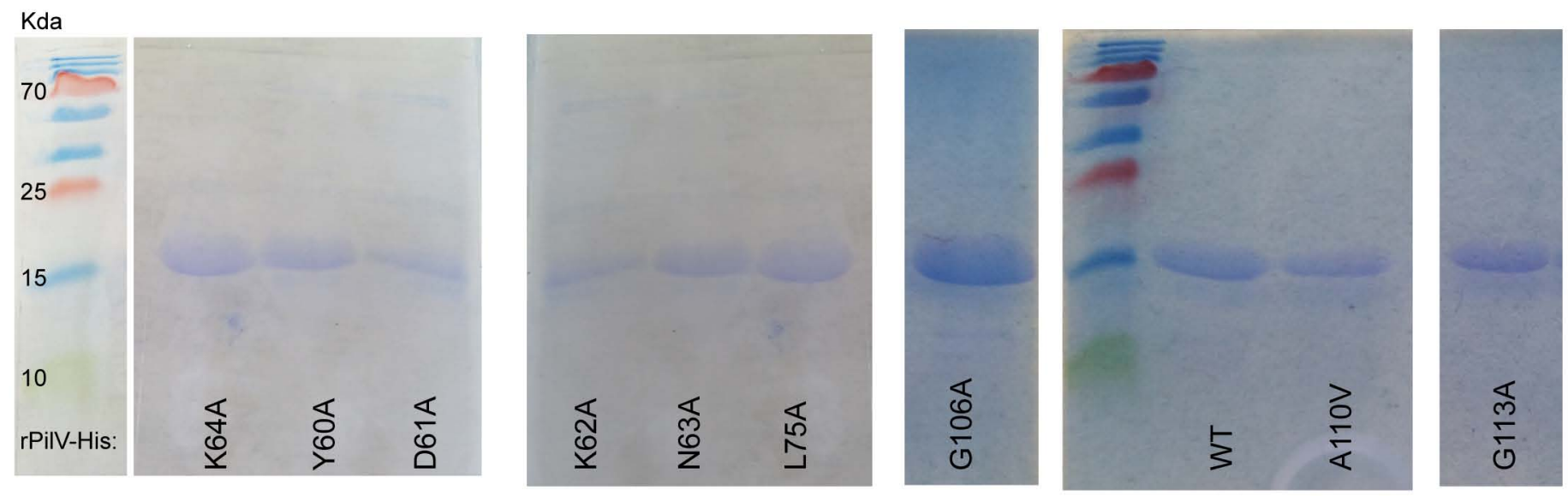


Supplemental figure 5

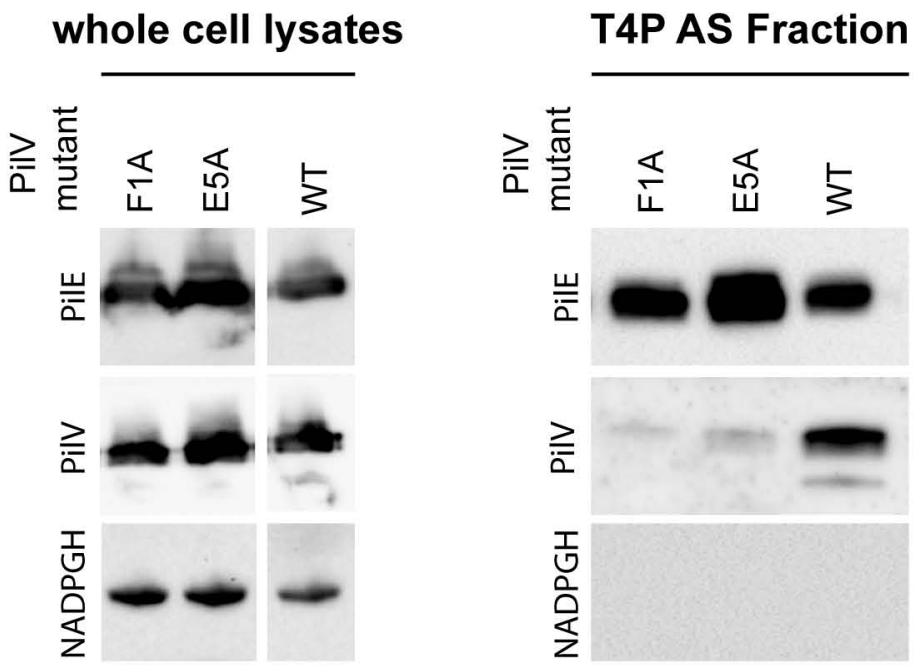


Supplemental figure 6

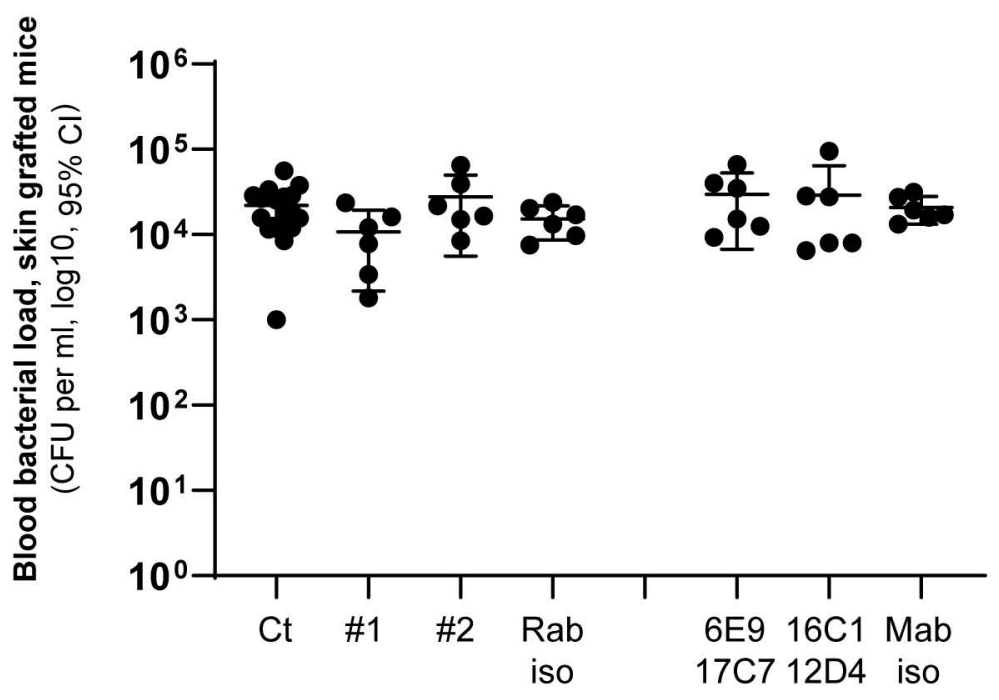


Table S2. Effect of PilV single amino acid substitutions on bacteria adhesion, recombinant pilin expression and inhibition of adhesion.

\begin{tabular}{|l|c|c|c|}
\hline Mutant & $\begin{array}{c}\text { Adhesion of } \\
\text { bacteria }^{1}\end{array}$ & $\begin{array}{c}\text { rPilV } \\
\text { variant } \\
\text { expression }\end{array}$ & $\begin{array}{c}\text { Inhibition } \\
\text { of } \\
\text { adhesion }\end{array}$ \\
\hline WT & $++{ }^{3}$ & + & +++ \\
\hline$\Delta$ pilV & $+/-$ & NA & NA \\
\hline Y60A & - & + & - \\
\hline D61A & - & + & - \\
\hline K62A & - & + & ++ \\
\hline N63A & - & + & + \\
\hline K64A & $+/-$ & + & $+/-$ \\
\hline L65A & - & NO & NO \\
\hline L75A & $+/-$ & + & - \\
\hline P91A & - & NO & NO \\
\hline D95A & - & NO & NO \\
\hline G106A & $+/-$ & + & - \\
\hline G107A & - & NO & NO \\
\hline A110V & $+/-$ & + & $+/-$ \\
\hline G113A & - & + & +++ \\
\hline
\end{tabular}

${ }^{1}$ Adhesion: $\%$ of of pil $V_{\mathrm{WT}}$ complemented strain

${ }^{2}$ Inhibition of $N$. meningitidis binding to hCMEC/D3 cells

${ }^{3}++=100 \% ;+/-<20 \% ;-<1 \%$

$\mathrm{NA}=$ not applicable

$\mathrm{NO}=$ not obtained 
Table S3. Bacterial strains, plasmids and primers

\begin{tabular}{|c|c|c|}
\hline Reagent & Description & Source/reference \\
\hline \multicolumn{3}{|l|}{ Strains } \\
\hline $\begin{array}{l}\text { E. coli } \mathrm{SHuffle} \mathrm{e}^{\circledR} \mathrm{T} 7 \\
\text { Express } \text { lys } Y \text { Competent } \\
\text { E. coli cells }\end{array}$ & 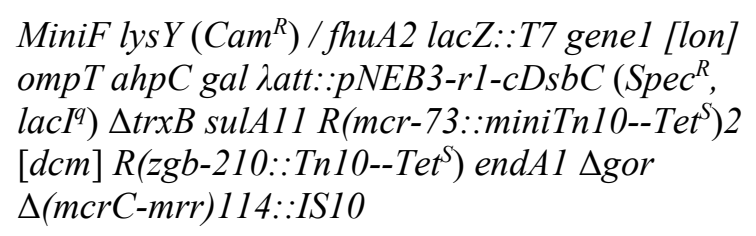 & $\begin{array}{l}\text { New England } \\
\text { Biolabs }\end{array}$ \\
\hline E. coli BL21 (DE3) & F- ompT hsdSB (rBmB-) gal dcm (DE3) & $\begin{array}{l}\text { Thermo Fisher } \\
\text { Scientific }\end{array}$ \\
\hline $\begin{array}{l}\text { N. meningitidis strain } 8013 \\
\text { Wild type, } \Delta \text { pilE \& } \Delta \text { pilV }\end{array}$ & $\begin{array}{l}\text { piliated capsulated Opa- Opc- variant of the } \\
\text { serogroup C meningococcal clinical isolate } \\
\text { NEM } 8013\end{array}$ & $\begin{array}{l}\text { (Bernard et al., } \\
\text { 2014) }\end{array}$ \\
\hline
\end{tabular}

\section{Plasmids}

\begin{tabular}{|c|c|c|}
\hline pET15b & $\begin{array}{l}\text { Encodes an N-terminal His•Tag }{ }^{\circledR} \text { sequence } \\
\text { followed by a thrombin site, } \mathrm{Ap}^{\mathrm{R}} \text {, T7lac }\end{array}$ & Novagen \\
\hline pET15b-pilV & $\begin{array}{l}\text { Encodes PilV (residues 29-122) with an N-terminal } \\
\text { His-tag and a thrombin cleavage site }\end{array}$ & This study \\
\hline pET22b & $\begin{array}{l}\text { Carries an N-terminal pelB signal sequence for } \\
\text { potential periplasmic localization, plus optional C- } \\
\text { terminal His } \bullet \text { Tag sequence }\end{array}$ & Novagen \\
\hline pET22b-pilV & $\begin{array}{l}\text { Encodes PilV (residues 29-122) with an C-terminal } \\
\text { His-tag }\end{array}$ & $\begin{array}{l}\text { (Virion et al., } \\
\text { 2019) }\end{array}$ \\
\hline pET22b-comP & $\begin{array}{l}\text { Encodes ComP (residues 29-149) with an C- } \\
\text { terminal His-tag }\end{array}$ & $\begin{array}{l}\text { (Virion et al., } \\
\text { 2019) }\end{array}$ \\
\hline
\end{tabular}

Primers used in this work

Complemented strain: pkh37-pilVFAM18 and phk37-pilVZ2491

Primers for structure study: pET15b-pilV

$\begin{array}{ll}\text { pilV-for } & \text { GCAATTCCATATGCGGCGCGTCCGCCTG TC } \\ \text { pilV-rev } & \text { CCGCGGATCCTTAGTCGAAGCCGGGGCAG }\end{array}$

Triple mutant: XXX->AAA 


\begin{tabular}{|c|c|}
\hline \multicolumn{2}{|c|}{ Mutants were obtained by PCR mutagenesis. Mutations are carried by the forward primer (FW) } \\
\hline Vmut_SCPGFD_117-122_rv & TAATTAATTAAGTACTATGCATGTCTAGA \\
\hline Vmut_GFD_fw & TGCGGCAGCGGGGCAGGATTGGTTTGTACC \\
\hline Vmut_SCP_fw & GTCGAAGCCTGCGGCAGCTTGGTTTGTACC \\
\hline Vmut_ASGTNQ_111-116_rv & TCCTGCCCCGGCTTCGAC \\
\hline Vmut_TNQ_fw & TGCGGCAGCACCGGAAGCGGCAATGGTAC \\
\hline Vmut_ASG_fw & TTGGTTTGTTGCGGCAGCGGCAATGGTAC \\
\hline Vmut_DGGTIA_105-110_rv & GCTTCCGGTACAAACCAATCC \\
\hline Vmut_TIA_fw & TGCGGCAGCACCGCCGTCGTTGAGCGTAAC \\
\hline Vmut_DGG_fw & GGCAATGGTTGCGGCAGCGTTGAGCGTAAC \\
\hline Vmut_CVVTLN_99-104_rv & GACGGCGGTACCATTGCCGCTTCCGG \\
\hline Vmut TLN fw & TGCGGCAGCAACGACGCAGGTTTCGCCGTCGTTGG \\
\hline Vmut_CVV_fw & GTTGAGCGTTGCGGCAGCGGTTTCGCCGTCGTTGG \\
\hline Vmut_TNDGET_93-98_rv & TGCGTCGTTACGCTCAACG \\
\hline Vmut_GET_fw & TGCGGCAGCGTCGTTGGTGGTGGGATTG \\
\hline Vmut_TND_fw & GGTTTCGCCTGCGGCAGCGGTGGGATTGGGGTC \\
\hline Vmut_ADPNPT_87-92_rv & ACCAACGACGGCGAAACC \\
\hline Vmut_NPT_fw & TGCGGCAGCGGGGTCGGCCTGAAGG \\
\hline Vmut_ADP_fw & GGTGGGATTTGCGGCAGCCTGAAGGGTAAAGTGGTCG \\
\hline Vmut_DHFTLQ_81-86_rv & GCCGACCCCAATCCCAC \\
\hline Vmut_TLQ_fw & TGCGGCAGCAAAGTGGTCGGGGCTGAC \\
\hline Vmut_DHF_fw & CTGAAGGGTTGCGGCAGCGGGGCTGACTTTGCTTAAG \\
\hline Vmut LSKVSP 75-80 rv & GACCACTTTACCCTTCAGGCC \\
\hline Vmut_VSP_fw & TGCGGCAGCTTTGCTTAAGGTAACGTTGAAATATTTGTTTTG \\
\hline Vmut_LSK_fw & GGGGCTGACTGCGGCAGCGGTAACGTTGAAATATTTGTTTTG \\
\hline Vmut_KYFNVT_69-74_rv & TTAAGCAAAGTCAGCCCCGAC \\
\hline Vmut_NVT_fw & TGCGGCAGCGAAATATTTGTTTTGTTTCAGTTTGTTTTTGTCG \\
\hline Vmut_KYF_fw & GGTAACGTTTGCGGCAGCGTTTTGTTTCAGTTTGTTTTTGTCG \\
\hline Vmut_NKLKQN_63-68_rv & AAATATTTCAACGTTACCTTAAGCAAAGTCAGC \\
\hline Vmut_KQN_fw & TGCGGCAGCCAGTTTGTTTTTGTCGTAGGTTTTAAACGTC \\
\hline Vmut_NKL_fw & GTTTTGTTTTGCGGCAGCTTTGTCGTAGGTTTTAAACG \\
\hline Vmut_FKTYDK_57-62_rv & AACAAACTGAAACAAAACAAATATTTC \\
\hline Vmut_YDK_fw & TGCGGCAGCGGTTTTAAACGTCCCTTTTTGG \\
\hline Vmut_FKT_fw & TTTGTCGTATGCGGCAGCCGTCCCTTTTTGG \\
\hline Vmut_YRQKGT_51-56_rv & TTTAAAACCTACGACAAAAACAAACTG \\
\hline Vmut_KGT_fw & TGCGGCAGCTTGGCGGTAGTAACGTTCC \\
\hline Vmut_YRQ_fw & CGTCCCTTTTGCGGCAGCGTAACGTTCCATGGTCTGC \\
\hline Vmut_ERY_48-50_rv & TACCGCCAAAAAGGGACG \\
\hline Vmut_ERY_fw & TGCGGCAGCCATGGTCTGCGCGTTG \\
\hline Vmut_TLLHNA_39-44_rv & CAGACCATGGAACGTTACTACC \\
\hline Vmut_HNA_fw & TGCGGCAGCCAGCAGGGTCGTCCTG \\
\hline Vmut_TLL_fw & CGCGTTGTGTGCGGCAGCCGTCCTGACTTCCGAC \\
\hline Vmut_TSEVRT_33-38_rv & ACCCTGCTGCACAACGC \\
\hline Vmut_VRT_fw & TGCGGCAGCTTCCGACAGGCGGACG \\
\hline Vmut TSE fw & CGTCCTGACTGCGGCAGCGCGGACGCGCC \\
\hline
\end{tabular}


Single mutant : $\mathrm{X}->\mathrm{A}$ (or A->V)

Mutants were obtained by PCR mutagenesis. Mutations are carried by the forward primer (FW)

SgMut_ASG_111-113 rv

TCCTGCCCCGGCTTCGAC

SgMut_G_113_fw

SgMut_S_112_fw

SgMut_A_111_fw

SgMut_TIA_108-110_rv

TTGGTTTGTtgcGGAAGCGGCAATGGT

TTGGTTTGTACCtgcAGCGGCAATGGT

TTGGTTTGTACCGGAtgcGGCAATGGT

GCTTCCGGTACAAACCAATCC

SgMut_A_110_fw

SgMut_I_109_fw

SgMut_T_108_fw

SgMut_DGG_105-107_rv

tgcAATGGTACCGCCGTCGTTGAGCGT

GGCtgcGGTACCGCCGTCGTTGAGCGT

GGCAATtgcACCGCCGTCGTTGAGCGT

SgMut_G_107_fw

SgMut_G_106_fw

SgMut_D_105_fw

SgMut_CVV_99-101_rv

SgMut_V_101_fw

SgMut_V_100_fw

SgMut_C_99_fw

SgMut_TND_93-95_rv

SgMut_D_95_fw

SgMut_N_94_fw

SgMut_T_93_fw

SgMut_NPT_90-92_rv

SgMut_T_92_fw

SgMut_P_91_fw

SgMut_N_90_fw

SgMut_TLQ_84-86_rv

SgMut_Q_86_fw

SgMut_L_85_fw

SgMut_T_84_fw

SgMut_LSK_75-77_rv

GCTTCCGGTACAAACCAATCC

GGCAATGGTtgcGCCGTCGTTGAGCGTA

GGCAATGGTACCtgcGTCGTTGAGCGTA

GGCAATGGTACCGCCtgcGTTGAGCGTA

GACGGCGGTACCATTGCCGC

GTTGAGCGTtgcGACGCAGGTTTCGCCGT

GTTGAGCGTAACtgcGCAGGTTTCGCCGT

GTTGAGCGTAACGACtgcGGTTTCGCCGT

TGCGTCGTTACGCTCAACG

GGTTTCGCCtgcGTTGGTGGTGGGATTGG

GGTTTCGCCGTCtgcGGTGGTGGGATTGG

GGTTTCGCCGTCGTTtgcGGTGGGATTGG

ACCAACGACGGCGAAACC

tgcGGGATTGGGGTCGGCCTGAAGGGTAA

GGTtgcATTGGGGTCGGCCTGAAGGGTAA

GGTGGGtgcGGGGTCGGCCTGAAGGGTAA

GCCGACCCCAATCCCAC

tgcAAGGGTAAAGTGGTCGGGGCTGACTT

CTGtgcGGTAAAGTGGTCGGGGCTGACTT

CTGAAGtgcAAAGTGGTCGGGGCTGACTT

GACCACTTTACCCTTCAGGCC

GGGGCTGACtgcGCTTAAGGTAACGTTGAA

GGGGCTGACTTTtgcTAAGGTAACGTTGAA

GGGGCTGACTTTGCTtgcGGTAACGTTGAA

SgMut_S_76_fw

SgMut_L_75_fw

SgMut_NKL_63-65_rv

AAATATTTCAACGTTACCTTAAGCAAAGTCAGC

SgMut_L_65_fw

SgMut_K_64_fw

SgMut_N_63_fw

SgMut_YDK_60-62_rv

SgMut_K_62_fw

SgMut_D_61_fw

SgMut_Y_60_fw

SgMut_E_1_fw

SgMut_E_1_rv

GTTTTGTTTtgcTTTGTTTTTGTCGTAGGTTTTAAACG

GTTTTGTTTCAGtgcGTTTTTGTCGTAGGTTTTAAACG

GTTTTGTTTCAGTTTtgcTTTGTCGTAGGTTTTAAACG

AACAAACTGAAACAAAACAAATATTTC

tgcGTCGTAGGTTTTAAACGTCCCTTTTTGG

TTTtgcGTAGGTTTTAAACGTCCCTTTTTGG

TTTGTCtgcGGTTTTAAACGTCCCTTTTTGG

GCCACGCTGCTCGAGCTGATGATTG

GCCTTTTTGAACGTTTTTCATAAAAT 
SgMut_F 5 fw

SgMut_F_5_rv

SgMut_L_65_fw

SgMut_L_65_rv

SgMut_K_66_fw

SgMut_K_66_rv

SgMut_K69_fw

SgMut_K69_rv

SgMut_Y_70_fw

SgMut_Y_70_rv
GCCCTGATGATTGCCGTCGCCATC

GAGCAGCGTAAAGCCTTTTTGAAC

GCCAAACAAAACAAATATTTCAACGTTACC

TTTGTTTTTGTCGTAGGTTTTAAACG GCCCAAAACAAATATTTCAACGTTACCTTA

CAGTTTGTTTTTGTCGTAGGTTTTA

GCCTATTTCAACGTTACCTTAAGCAAAGTC

GTTTTGTTTCAGTTTGTTTTTGTCGT

GCCTTCAACGTTACCTTAAGCAAAGTCAG

TTTGTTTTGTTTCAGTTTGTTTTTG 


\section{Supplemental Methods}

PilV expression for crystallization.

The pilV gene encoding residues 29-122 of PilV (rPilV) was cloned from $N$. meningitidis 8013 strain genomic DNA using primers pilV-for and pilV-rev (Table 1). PCR products were digested with NdeI and BamHI and ligated into pET15b (Novagen) digested with the same enzymes. The new plasmid, pET15b-pilV encoding PilV with an N-terminal hexahistidine tag (His-tag), was transformed into SHuffle ${ }^{\circledR}$ T7 Express lysS Competent E. coli cells (New England Biolabs). L-selenomethionine-substituted PilV was expressed by growing the bacterial cells in M9 minimal medium (3.4 mM Na2 $\left.\mathrm{HPO}_{4}, 2.2 \mathrm{mM} \mathrm{KH}_{2} \mathrm{PO}_{4}, 0.94 \mathrm{mM} \mathrm{NH}_{4} \mathrm{Cl}, 0.86 \mathrm{mM} \mathrm{NaCl}\right)$ supplemented with $0.2 \%$ glucose (w/v), $2 \mathrm{mM} \mathrm{MgSO}_{4}, 0.1 \mathrm{mM} \mathrm{CaCl}_{2}, 0.00005 \%$ thiamine (w/v) and $40 \mathrm{mg} / \mathrm{L}$ of all naturally occurring amino acids except Gly, Ala, Pro, Asn, Cys and Met shaking at $37{ }^{\circ} \mathrm{C}$ to an $\mathrm{OD}_{600}$ of 0.5 (Van Duyne et al., 1993). At this point, additional amino acids were added: Lys, Thr and Phe, $100 \mathrm{mg} / \mathrm{L}$; Leu, Ile and Val, $50 \mathrm{mg} / \mathrm{L}$; and L-SeMet, $60 \mathrm{mg} / \mathrm{L}$. PilV expression was induced with $0.4 \mathrm{mM}$ isopropyl $\beta$-D-1-thiogalactopyranoside (final concentration) and the culture was incubated for 5 hours at $37{ }^{\circ} \mathrm{C}$. Bacterial cells were pelleted by centrifugation at $5000 \mathrm{~g}$ for $30 \mathrm{~min}$ at $4{ }^{\circ} \mathrm{C}$. The cell pellet was washed and resuspended in Buffer $1(20 \mathrm{mM}$, Tris- $\mathrm{HCl}, \mathrm{pH} 8.0,100 \mathrm{mM} \mathrm{NaCl})$ containing $1 \mathrm{mg} / \mathrm{mL}$ lysozyme and EDTA-free protease inhibitor cocktail (Roche, 1 tablet/L original culture). The cells were incubated at room temperature for 2 hours then cooled on ice and lysed by sonication. The cell lysate was centrifuged at $7,000 \times \mathrm{g}$ for $20 \mathrm{~min}$ at $4{ }^{\circ} \mathrm{C}$ and the supernatant was discarded. The cell pellet containing insoluble PilV was resuspended in ice cold Buffer 2 (20 $\mathrm{mM}$ Tris-HCl, $\mathrm{pH} 8.0,0.5 \mathrm{M} \mathrm{NaCl}, 2 \%$ Triton $\mathrm{X}-100$ ) containing $2 \mathrm{M}$ urea and the sonication and centrifugation steps were repeated. The resulting pellet was suspended in ice cold Buffer 2 and centrifuged at $7,000 \mathrm{x}$ for $20 \mathrm{~min}$ at $4{ }^{\circ} \mathrm{C}$. The pellet was resuspended in Buffer $3(20 \mathrm{mM}$ 
Tris- $\mathrm{HCl} \mathrm{pH}$ 8.0, $0.5 \mathrm{M} \mathrm{NaCl}, 5 \mathrm{mM}$ imidazole) containing $6 \mathrm{M}$ guanidine hydrochloride and stirred for an hour at room temperature. The solution was centrifuged at 7,000 x $g$ for 15 min at $4{ }^{\circ} \mathrm{C}$ and the supernatant was passed through $0.4 \mu \mathrm{m}$ filter to remove thin particles and insoluble aggregates. The filtered supernatant was loaded onto a Ni-NTA column (GE Healthcare Life Sciences) and the column with bound denatured His-PilV was washed with 5 column volumes of Buffer 3 containing $6 \mathrm{M}$ guanidine hydrochloride. Next, the column was washed with 5 column volumes of Buffer 4 (20 mM Tris- $\mathrm{HCl}, \mathrm{pH}$ 8.0, $0.5 \mathrm{M} \mathrm{NaCl}, 20 \mathrm{mM}$ imidazole, $1 \mathrm{mM}$ 2-mecaptoethanol) containing $6 \mathrm{M}$ urea followed by 5 column volumes of Buffer 4 containing $3 \mathrm{M}$ urea and 5 column volumes of Buffer 4 alone. The denatured and refolded His-PilV was eluted with Buffer $5(20 \mathrm{mM}$ Tris- $\mathrm{HCl}$ pH 8.0, 0.5 M NaCl, $250 \mathrm{mM}$ imidazole). Where appropriate, thrombin was added to the protein $(1 \mathrm{U} / \mathrm{mg})$, to cleave off the N-terminal His tag and the solution was dialyzed against Buffer 1. PilV was concentrated to 8 $\mathrm{mg} / \mathrm{mL}$ using an Amicon stirred cell concentrator (Millipore) and benzamidine was added to a final concentration of $2 \mathrm{mM}$. The protein was cryocooled in liquid nitrogen and stored at -80 ${ }^{\circ} \mathrm{C}$.

\section{Recombinant expression of His-tagged PilV and His-tagged ComP}

Recombinants PilV (residues 29-122) and ComP (residues 29-149), both with a C-terminal hexahistidine tag (rPilV-His, rComP-His) were produced as periplasm-directed proteins using the pET22b plasmid (pET22 $\beta$-pilV and pET22 $\beta$-comP) as described previously (Virion et al., 2019). Plasmids were used to transform E. coli BL21 (DE3) (Thermo Fisher Scientific) and bacteria were grown for 3 hours at $30^{\circ} \mathrm{C}$ in lysogeny broth (LB) followed by 16 hours at $16{ }^{\circ} \mathrm{C}$ in LB supplemented with $1 \mathrm{mM}$ isopropyl $\beta$-D-1-thiogalactopyranoside. rPilV-His and rComPHis were extracted from the periplasm and loaded onto a Ni-NTA column (Thermo Scientific). The His-tagged pilin was eluted using elution buffer $\left(50 \mathrm{mM} \mathrm{NaH}_{2} \mathrm{PO}_{4}, \mathrm{pH} 8,300 \mathrm{mM} \mathrm{NaCl}\right.$, 
$250 \mathrm{mM}$ imidazole) and the buffer was exchanged with phosphate-buffered saline (PBS) using an Amicon $10 \mathrm{kDa}$ molecular weight cut-off (MWCO) membrane (Merck Millipore) to a concentration of $1 \mathrm{mg} / \mathrm{mL}$. The purity of His-tagged pilins was assessed by sodium dodecyl sulfate-polyacrylamide gel electrophoresis (SDS-PAGE) and Coomassie blue staining (Supplemental Fig. 4E). rPilV variants were generated by PCR mutagenesis of pilV on pET22bpilV with the mutagenesis primers used for pilV mutants expressed in the $\Delta$ pilV strain (Supplemental table S3).

\section{Scanning mutagenesis of N. meningitidis pilV}

Mutations were introduced into the pilV gene inserted in plasmid pKH37 between the $N$. meningitidis genes lctP and $a s p C$ and downstream of the lacP promoter (Ramsey et al., 2012). These mutations substituted amino acid triplets with alanine triplets (or valine when the WT amino acid is alanine) throughout the globular domain, from residue 33 to 122 . The plasmids were used to transform the PilV deficient $N$. meningitidis strain $2 \mathrm{C} 4.3(\Delta V)$, whereby recombination occurs between the plasmid-borne pilV mutant allele and the genome between lctP and $a s p C$ and pilV is expressed under control of the lacP promoter (Ramsey et al., 2012). To examine the impact of the alanine substitutions, complemented $\Delta V$ mutants were first tested for their ability to incorporate PilV into the pili by shearing the pili from the bacterial surface, concentrating them using ammonium sulfate precipitation and assessing PilV levels by SDSPAGE and immunoblotting (Fig. SM1). Mutants expressing PilV in surface pili were tested for their ability to adhere to hCMEC/D3 endothelial cells (Fig. SM1). Of these mutants, several show profound adhesion defects comparable to that of the uncomplemented $\Delta V$ mutant: $60-62$, 63-65, 75-77, 90-92, 93-95, 99-101, 105-107, 108-110, 111-113.

To identify individual residues involved in adhesion, we generated a panel of single amino-acid 
alanine substitutions and expressed these pilV mutants ectopically in $N$. meningitidis $\Delta V$. PilV levels in sheared pili and adherence to endothelial cells were tested as for the triple mutants (Fig. SM2). Of particular interest are the complemented strains that expressed mutant PilV in their sheared pilus fractions yet show little or no adhesion: mutants spanning residues 60-64 as well as L75A, G106A, G107A, A110V and G113A. These residues may be directly involved in host cell adhesion.

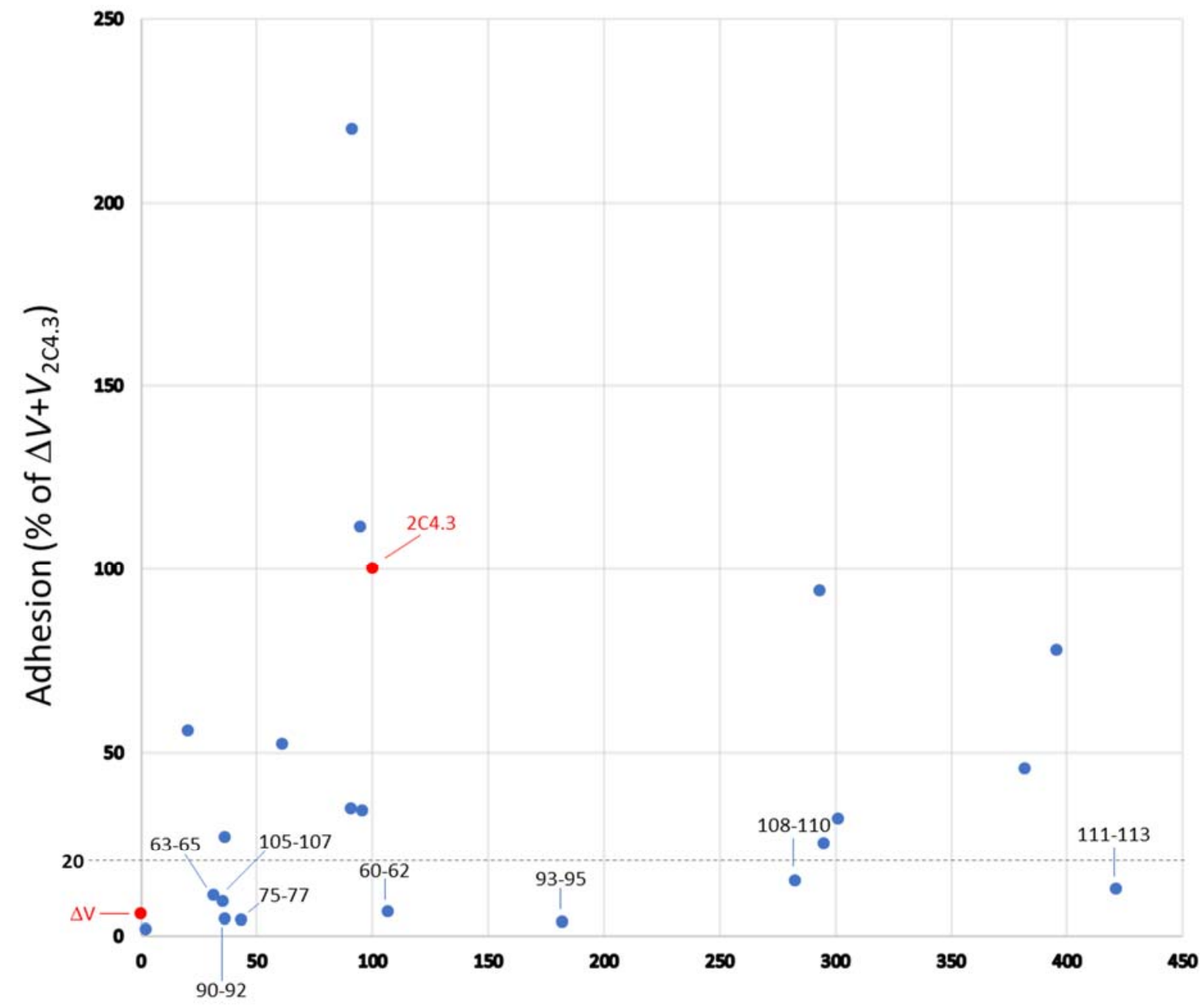

PilV expression in sheared pili (Intensity in Western Blot; \% of $\Delta V+V_{2 c 4.3}$ )

Figure SM1. Analysis of pilus-mediated adhesion to endothelial cells for pilV triple mutants. 
Adhesion of $\Delta V$ strain and $\Delta V$ strains complemented with wild-type $N$. meningitidis pilV or pilV triple mutants. Human endothelial cells (hCMEC/D3) were infected with pilV-complemented strains for 30 minutes and the number of cell-associated bacteria was determined by counting CFUs. Adhesion is expressed as mean of CFUs normalized on mean of CFUs for the strain expressing wild type pilV from two independent experiments. PilV expression in sheared pili was assessed by immunoblots of sheared ammonium sulfate-precipitated pili from $N$. meningitidis $\Delta V$ complemented with pilV mutants and expressed as a percentage of the density of the PilV band for the strain expressing wild type sequence of pilV $\left(\Delta V+V_{2 C 4.3}\right)$.

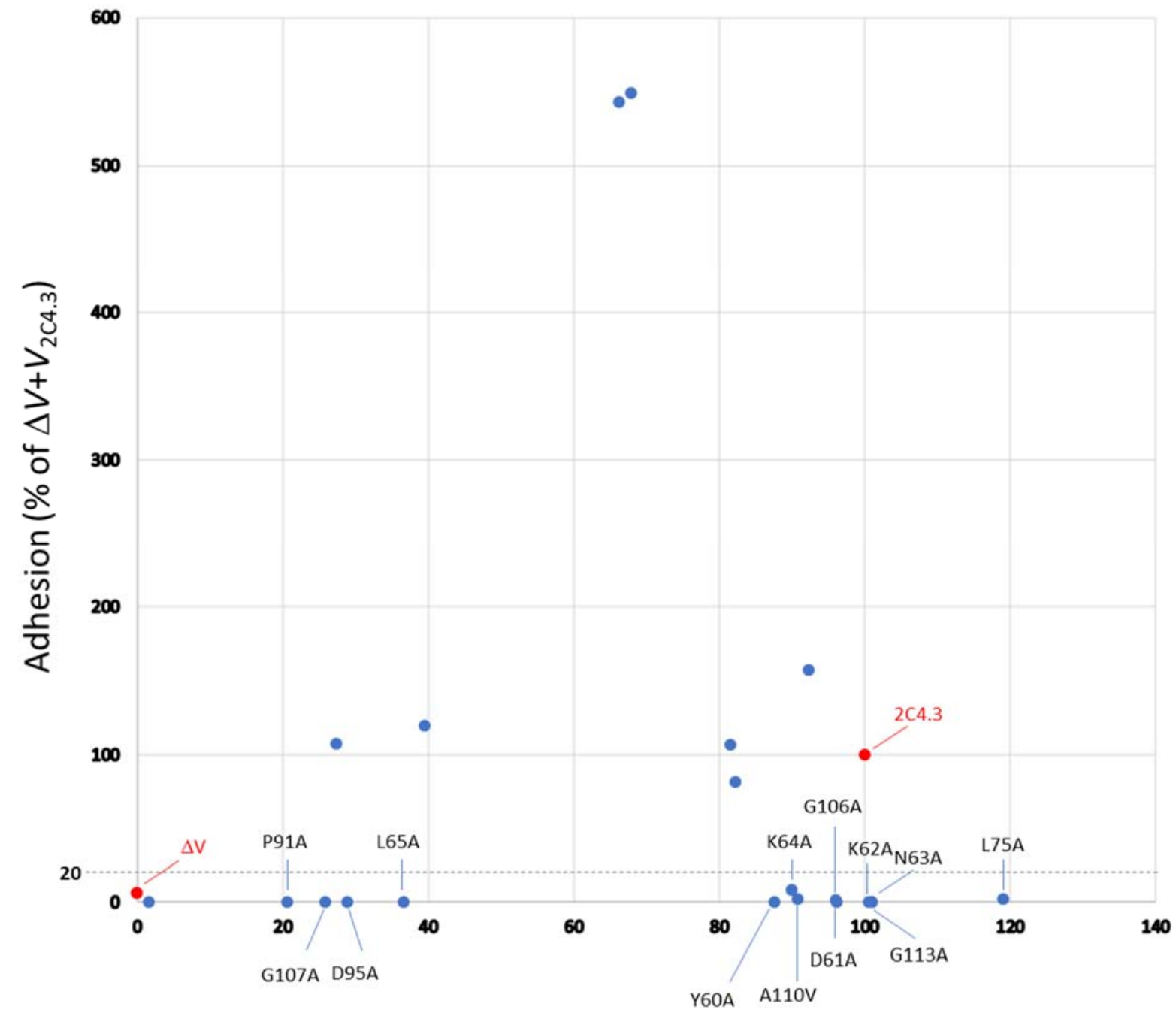

PilV expression in sheared pili (Intensity in Western Blot; \% of $\Delta V+V_{2 c 4.3}$ )

Figure SM2. Analysis of pilus-mediated adhesion to endothelial cells for pilV single mutants. 
Adhesion of $\Delta V$ strain and $\Delta V$ strains complemented with wild-type N. meningitidis pilV or pilV single mutants. Human endothelial cells (hCMEC/D3) were infected with pilV-complemented strains for 30 minutes and the number of cell-associated bacteria was determined by counting CFUs. Adhesion is expressed as mean of CFUs normalized on mean of CFUs for the strain expressing wild type sequence of pilV for two independent experiments. PilV expression in sheared pili was assessed by immunoblots of sheared ammonium sulfate-precipitated pili from pilV-complemented strains, and expressed as percentage of PilV band intensity for the strain expressing wild type sequence of pil $\left(\Delta V+V_{2 C 4.3}\right)$.

\section{Reference:}

Ramsey, M.E., Hackett, K.T., Kotha, C., Dillard, J.P., 2012. New Complementation Constructs for Inducible and Constitutive Gene Expression in Neisseria gonorrhoeae and Neisseria meningitidis. Appl. Environ. Microbiol. 78, 3068-3078. https://doi.org/10.1128/AEM.0787111 